\title{
Laser Applications of Transparent Polycrystalline Ceramic
}

\author{
Qihong Lou, Jun Zhou, Yuanfeng Qi and Hong Cai \\ Shanghai Institute of Optics and fine mechanics \\ P.R.China
}

\section{Introduction}

High power lasers are widely used in a variety of applications, including materials processing, remote sensing, free-space communications, laser particle acceleration, gravitational wave interferometers, and even inertial confinement fusion (ICF) [1]. The optical gain media of the system is the key factor for efficient laser oscillation. Since Maiman discovered the first ruby laser in 1960, numerous materials have been developed and improved to achieve high efficiency and high power for all-solid-state lasers. There are three primary groups of solid state host materials: single crystals, glasses and ceramics. Among them Nd:YAG single crystal may be the most widely used laser media. But Nd:YAG single crystal grown by conventional Czochralski method has its own insurmountable disadvantages such as expensive, time-consuming, small size and low concentration [2], which has limited its applications in high power lasers. And for Nd-doped glass material, though it is very easy to get large size and high concentration, but its thermal conductivity and gain are quite low and the laser efficiencies were not satisfying. Polycrystalline ceramics is an aggregate of crystalline grains, each randomly oriented with respect to neighboring grains. Since the 1960s, it has been speculated that a dense polycrystal of an isotropic, pure material would be optically indistinguishable from a single crystal of the same material. The only problem has been finding a fabrication method. Now materials scientists in Japan have come up with a way to mass-produce polycrystalline ceramics materials that maintain high conversion efficiency and good optical characteristics as well as single crystals. Further more, the ceramics laser materials manufacturing method has five distinct advantages over single-crystal growth:

- Ease of manufacture: It takes 4-6 weeks to grow crystals using the Czochralski method, but thes method makes rods in just a few days.

- Less expensive: Single crystals have to be grown in an expensive iridium crucible. Ceramics rod growth requires no crucible and is also faster. The cost of a single crystal increases dramatically with its size, unlike ceramics.

- Fabrication of large size and high concentration laser medium: Size of rods Singlecrystal growth limits crystal size, which in turn limits the potential output power. The maximum crystal size is about $23 \mathrm{~cm}$ long and the $\mathrm{Nd}^{3+}$ doping concentration is no more than 2 at. $\%$. But polycrystalline ceramic YAG can be made as large as $1 \mathrm{~m} \times 1$ $\mathrm{m} \times 0.02 \mathrm{~m}$ and up to 4 at. $\%$ doping with no gradient. 
- Multi-layer and multi-functionality Ceramics structure: Ceramics fabrication could enable the incorporation of Q-switching and Raman shifting within the source, which is impossible with a single crystal.

- Mass-production: Suitability Ceramics materials can be fabricated in a production-line fashion, reducing the time and cost required for single-crystal YAG rod manufacturing.

\section{The development of transparent ceramics (history)}

Since 1960 's, a number of researchers had speculated that a theoretically dense polycrystal of an isotropic, pure material would be optically indistinguishable from a single crystal of the same material. In 1966, Hot-pressed CaF2 doped dysprosium appears to be the first reported polycrystalline material which established laser oscillation [4]. Then several decades passed, no remarkable development had been acquired. The problem with making laser materials from ceramics material is that ceramics are polycrystalline and some of their characteristics, such as grain boundaries, pores, composition gradients and lattice imperfections, increase the scattering of light in the host. This adds to the opacity of the material, making it unsuitable for laser action. The key is to find a manufacturing method in which the crystals that make up the rod are very similar in size and small enough to have little effect on incident light with a wavelength of around $1 \mu \mathrm{m}$. Only in the last decade have ceramics laser materials received much attention, after manufacturing breakthrough coming with highly transparent nanocrystalline YAG doped with $\mathrm{Ln}^{3+}$ activators, in particular $\mathrm{Nd} 3+$ ions. In 1995, the first Nd:YAG ceramics laser was developed by Akio Ikesue and colleagues at Japan's Krosaki Corporation. Ikesue used a hot-press method to make the ceramics laser materials. [5] Later in 1999, another research team led by Ken-ichi Ueda improved Nd:YAG ceramics successfully by combining liquid-phase chemical reaction with vacuum sintering technique to produce the similarly-sized nanoparticles for ceramics formation. The nanoparticles are homogenous, so any pressure need not use. [6-7]. High quality, high transparent Nd:YAG ceramics with much low scattering losses have been fabricated. Optical absorption, fluorescence and emission spectra, physical and laser properties of Nd: YAG ceramics have been measured and compared with those of Nd: YAG single crystals, and almost identical superiority features have been obtained in qualitative analysis [8-10]. It shows that Nd:YAG ceramics are indeed potential superexcellent gain media for high efficient and high power lasers. Using these Nd:YAG ceramics samples, high slope efficiency of $68 \%$ was achieved under end-pumping disk laser [11]. And for high power laser oscillation, output power from $499 \mathrm{~mW} \rightarrow 31 \mathrm{~W} \rightarrow 72 \mathrm{~W} \rightarrow 88 \mathrm{~W} \rightarrow 128 \mathrm{~W} \rightarrow 1460 \mathrm{~W}$ were reported one by one [12-15]. Now Nd:YAG ceramics slabs for solid-state heat capacity laser were reported hit $67 \mathrm{~kW}$ high power. In order to suppress parasitic oscillation, Sm:YAG ceramics was fabricated as for an absorber, and its optical properties were investigated. Higher power ceramics laser is still-evolving. The biggest advantage is the scaling to the meter-size plate. As a result, the ceramics laser is the most promising active medium for the laser fusion drivers.

Making ceramics YAG crystals is not restricted to neodymium-doped material or YAG crystals. $\mathrm{Er}^{3+}, \mathrm{Yb}^{3+}, \mathrm{Nd}^{3+}, \mathrm{Eu}^{3+}, \mathrm{Dy}^{3+}$ and $\mathrm{Cr}^{4+}$ as well as Sesquioxide host crystals can be made also. $\mathrm{Nd}: \mathrm{Y}_{2} \mathrm{O}_{3}$ and $\mathrm{Yb}: \mathrm{Y}_{2} \mathrm{O}_{3}$ ceramics laser materials as having an extra advantage over single crystals. It is very hard to grow a single $\mathrm{Y}_{2} \mathrm{O}_{3}$ crystal because its melting temperature is $2430{ }^{\circ} \mathrm{C}$. The sintering temperature for $\mathrm{Y}_{2} \mathrm{O}_{3}$ is some $700{ }^{\circ} \mathrm{C}$ lower than its melting point, 
meaning that large $\mathrm{Y}_{2} \mathrm{O}_{3}$ ceramics could be manufactured using a vacuum sintering method. One of the advantages of $\mathrm{Y}_{2} \mathrm{O}_{3}$ is its thermal conductivity, which is twice that of YAG for ceramics materials. This could make it more appropriate for using in the femto-second lasers for industry. Ceramics Y2O3 laser generated Sub-200 fs Fourier-limited pulses in the SESAM mode locking.

Another possibility is that ceramics laser rods could incorporate multiple-laser functionality. All ceramics passively Q-switched Yb:YAG/Cr4+:YAG microchip laser with shortest pulse width of 380 ps has been achieved.

The still developing Nd:YAG ceramics are very good alternative to Nd:YAG single crystals for high energy pulse laser applications in the near future.

\section{Comparison of laser characteristics between transparent ceramics and single crystal}

\subsection{Spectroscopic analysis}

Optical absorption and emission measurements were carried out as follows. The normalized intensity of room temperature absorption spectrum of 1at.\% Nd:YAG ceramics and 1.1at.\% Nd:YAG single crystal is shown in Figure. 1(a). From this figure, we see that the main absorption peak of $2 \%$ ceramics is centered at $808.56 \mathrm{~nm}$ which is slightly red shifted compared to that of single crystal $\sim 808.48 \mathrm{~nm}$ ! Because of a slight change in the crystal field in the high neodymium concentration samples. Figure. 1(b) shows the room temperature fluorescence spectra for 1at.\% Nd:YAG ceramics and 1.1at.\% Nd:YAG single crystal, respectively. For comparison, the fluorescence spectrum for single crystal and ceramics are normalized and put together. A slight redshift was also observed in emission spectrum because of high neodymium concentration. The emission peak of Nd:YAG ceramics is centered at $1064.2 \mathrm{~nm}$ which is $0.1 \mathrm{~nm}$ redshifted away from that of Nd:YAG single crystal. Except the slight redshift, the two spectra are almost identical to each other.

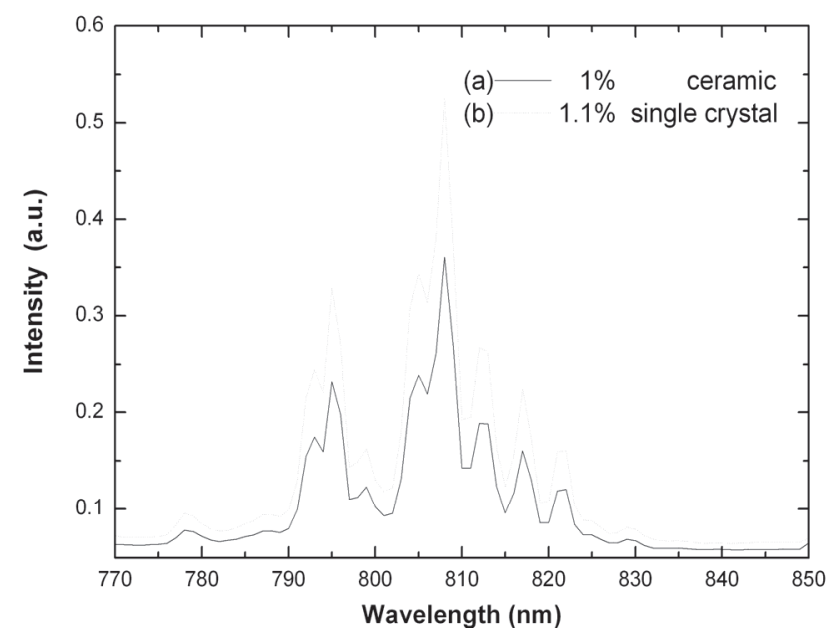

Fig. 1. (a). Comparison of room-temperature absorption spectrum from $770 \mathrm{~nm}$ to $850 \mathrm{~nm}$ between Nd:YAG ceramic and single crystal. 


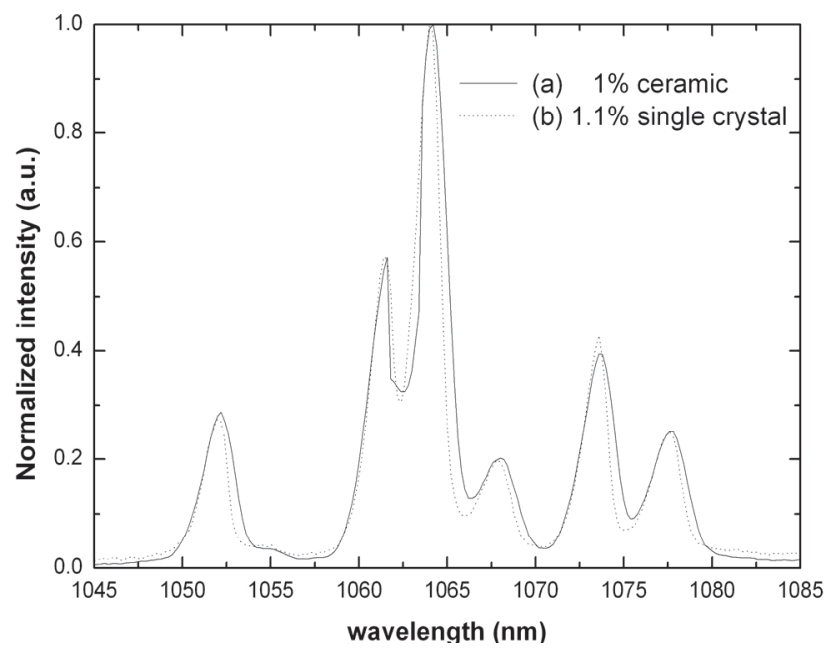

Fig. 1. (b). Comparison of room-temperature fluorescence spectrum from 1045 to $1085 \mathrm{~nm}$ between Nd:YAG ceramic and single crysPulse trains from Q-switched ceramic

\subsection{Fluorescence lifetime}

As reported by Konoshima Chemical, Co., Ltd. and Ueda's research group, [16] the fluorescence lifetime for single crystal and ceramics have been obtained through curve fitting on the fluorescence decay curve. The fluorescence lifetime of Nd:YAG ceramics and single crystal versus neodymium concentration. The fluorescence lifetime for $0.6 \% \mathrm{Nd}$ :YAG single crystal and $0.9 \% \mathrm{Nd}$ :YAG single crystal are $256.3 \mu \mathrm{s}$ and $248.6 \mu \mathrm{s}$, respectively( Figure. 2), which agrees well with the earlier reports [17]. Fluorescence lifetimes of $257.6 \mu \mathrm{s}$, $237.6 \mu \mathrm{s}, 184.2 \mu \mathrm{s}$ and $95.6 \mu \mathrm{s}$ have been measured, respectively, for $0.6 \%, 1 \%, 2 \%$ and $4 \%$ Nd:YAG ceramics. These data also agree well with the results in [17]. The fluorescence

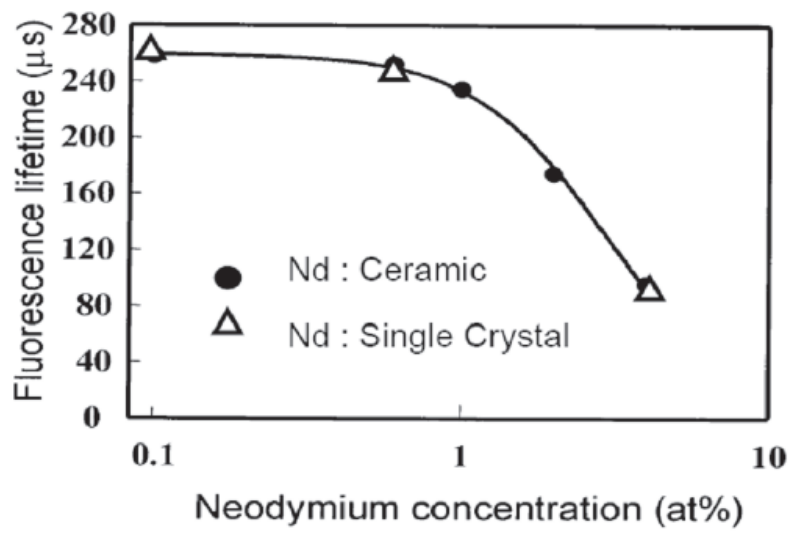

Fig. 2. Fluorescence lifetime of Nd:YAG ceramics and single crystal versus neodymium concentration. Solid line is the fitted curve for ceramics fluorescence lifetime. 
lifetime decreases dramatically when neodymium concentration exceeds $1 \%$. The fluorescence lifetimes for $0.6 \%$ doped single crystal and ceramics are almost identical (only $1.3 \mu$ difference). The fluorescence lifetime difference between $0.9 \% \mathrm{Nd}$ :YAG single crystal and $1 \% \mathrm{Nd}$ :YAG ceramics is $11 \mu \mathrm{s}$. It can be predicted that for the same concentration of Nd:YAG single crystal and ceramics, for example, $0.9 \%$ concentration, the lifetime difference should be less than $11 \mu \mathrm{s}$. From the fitted curve for ceramics fluorescence lifetime, the lifetime for $0.9 \% \mathrm{Nd}$ :YAG ceramics is $244.2 \mu \mathrm{s}$, which is only $4.4 \mu \mathrm{s}$ different from that of $0.9 \% \mathrm{Nd}$ :YAG single crystal. It indicated that the neodymium ions inside the grain have the same conditions as those of single crystal, and the fluorescence lifetime difference is caused only by the neodymium ions in the vicinity of grain boundaries.

The wavefront distortion picture of a single crystal YAG slab and ceramics YAG slab near the facet part measured by a Zygo interferometer is show in Figure. 3. From this figure, one can see that near the facet part, the wavefront was seriously distorted for the single crystal YAG. But for a ceramics Nd : YAG slab, because there is no facet problem, the wavefront distortion picture (right) shows a homogeneous pattern, which is much better than that of a single crystal. A crystalline YAG has poor optical homogeneity because of its facet structure during growing process. The optical homogeneity of ceramics YAG is good as well as glass.

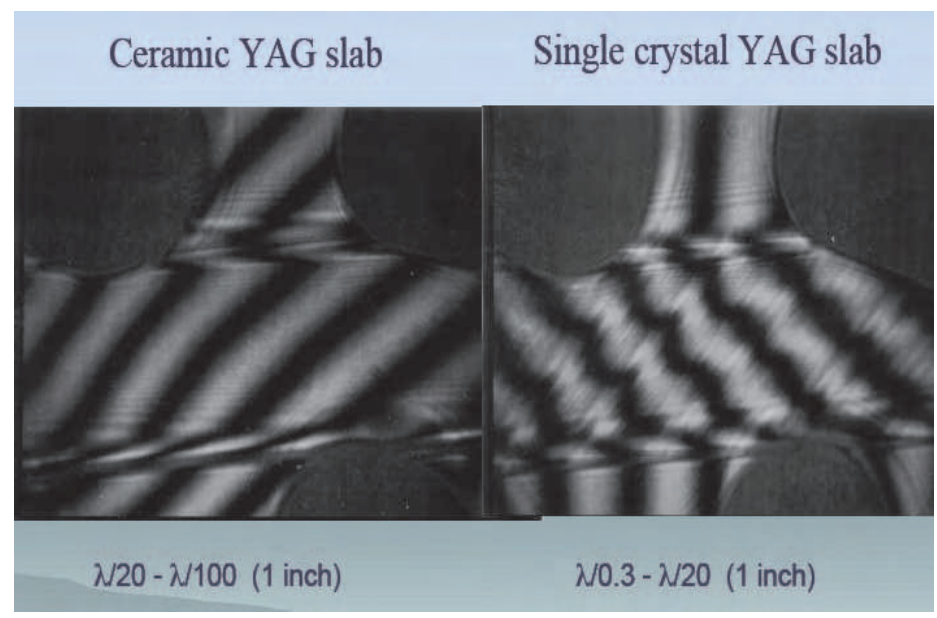

Fig. 3. The wavefront distortion picture of a single crystal YAG and ceramics YAG slab

\section{High efficiency Nd: YAG ceramics laser}

\subsection{Quasi-CW Nd:YAG ceramics laser}

By using a quite uniformly side-around arranged compact pumping system, A high efficiency high power quasi-CW laser with a Nd:YAG ceramics rod has been demonstrated. With $450 \mathrm{~W}$ quasi-CW stacked laser diode bars pumping at $1064 \mathrm{~nm}, 236 \mathrm{~W}$ optimum output laser at $1064 \mathrm{~nm}$ was obtained. The optical-to-optical conversion efficiency was $52.5 \%$ and corresponding slope efficiency was $62 \%$.

A schematic diagram of the laser setup is shown in Figure. 4. The Nd:YAG ceramics rod used in the experiment was $75 \mathrm{~mm}$ in length and $5 \mathrm{~mm}$ in diameter with neodymium 
doping level of 1 at.\%. Both the end facets of the rod were flat and antireflection coated at $1064 \mathrm{~nm}$ in order to reduce the intra-cavity losses, and the lateral surface was frosted. The rear mirror of the laser cavity was high-reflection mirror at $1064 \mathrm{~nm}$ and a series of output coupling mirrors were prepared with reflectivity from $30 \%$ to $84 \%$ at $1064 \mathrm{~nm}$. Thus we could find the optimized output in experiment. The cavity length was about $195 \mathrm{~mm}$. The pump source was operated at $808 \mathrm{~nm}$. Liquid cooling was employed to remove heat from the ceramics rod and diode heat sink. The operation temperature was kept at about $16{ }^{\circ} \mathrm{C}$.

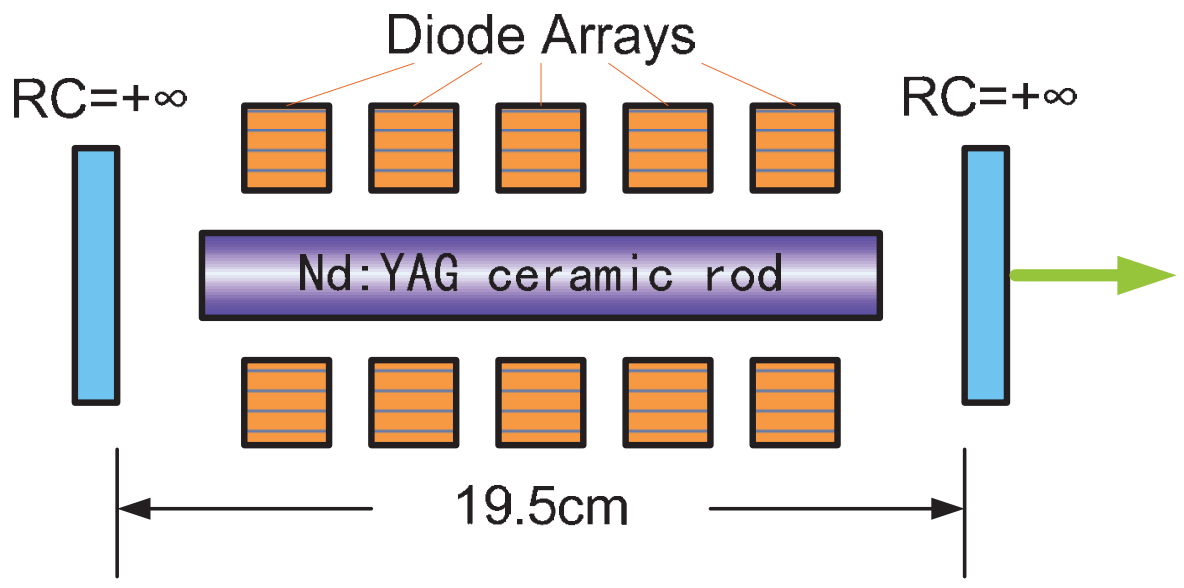

Fig. 4. Schematic diagram of experimental setup for side-pumped Nd:YAG ceramics rod laser.

In order to optimize the uniformity and radial profile of the pump distribution within the gain medium and decrease the coupling losses, we designed a compact side-around arranged direct radial-pumping head, of which cross-section configuration was illustrated in Figure. 5. The optical pump head consisted of nine LD stacked arrays mounted around the rod from 9 directions with proportional angle. The ceramics rod was mounted inside a flow-tube. The side-face of the ceramics rod and the emitting surface of the laser diodes were close proximity, and no coupling optics was employed between them. The coupling efficiency was by far the most desirable. Each LD stacked array consisted of five quasi-CW types LD bars, which were placed along the length of the laser rod and pumped perpendicularly to the direction of propagation of the laser radiation. Each bar generated 60 $\mathrm{W}$ peak powers. The arrays operating at $20 \%$ duty cycle were pulsed at a repetition rate of 1 $\mathrm{kHz}$ with a pulse width of $200 \mu \mathrm{s}$. The design of 9 LD arrays arranged around the ceramics rod symmetrical allowed optimizing the uniformity and radial profile of the pump distribution within the gain medium with good spatial overlap between pump radiation and low-order modes in the resonator, which in turn leads to a high-brightness laser output. Figure. 6 showed the 2D contour plot of pump intensity distribution simulated by computer with ray tracing method. 


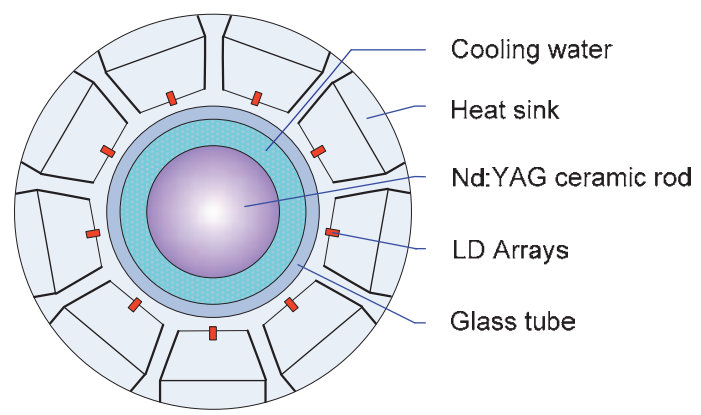

Fig. 5. Cross-section of large diode arrays compact side-pumped Nd:YAG ceramics laser head.

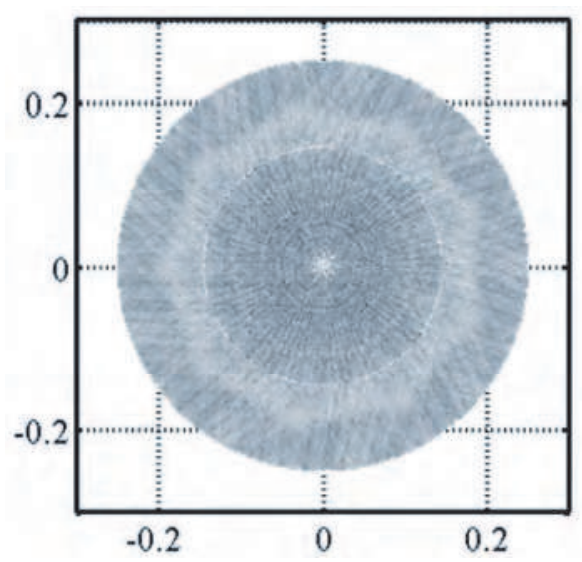

Fig. 6. 2D Contour plot of pump intensity distribution simulated by computer with ray tracing method.

By changing the rear mirror with different reflectivity of $30 \%, 50 \%, 62.5 \%, 78 \%$, and $83.4 \%$, we get a relationship laser output power as a function of the average pumping power, which was shown in Figure. 7. The output power increased almost linearly with the pumping power, and the optimum output appeared with the coupling mirror of the reflectivity near $78 \%$. When the pump current rose to $60 \mathrm{~A}$, the total average pump power was about $450 \mathrm{~W}$, and the maximum average power of $236 \mathrm{~W}$ multi-mode laser output was obtained by using optimum output coupling mirror. The optical-to-optical conversion efficiency was as high as $52.5 \%$ and corresponding slope efficiency was $62 \%$. No obvious evidence of saturation was observed from the output curve, which means higher output power is possible if higher pump power is available. It also indicated that the laser cavity is stable enough. 


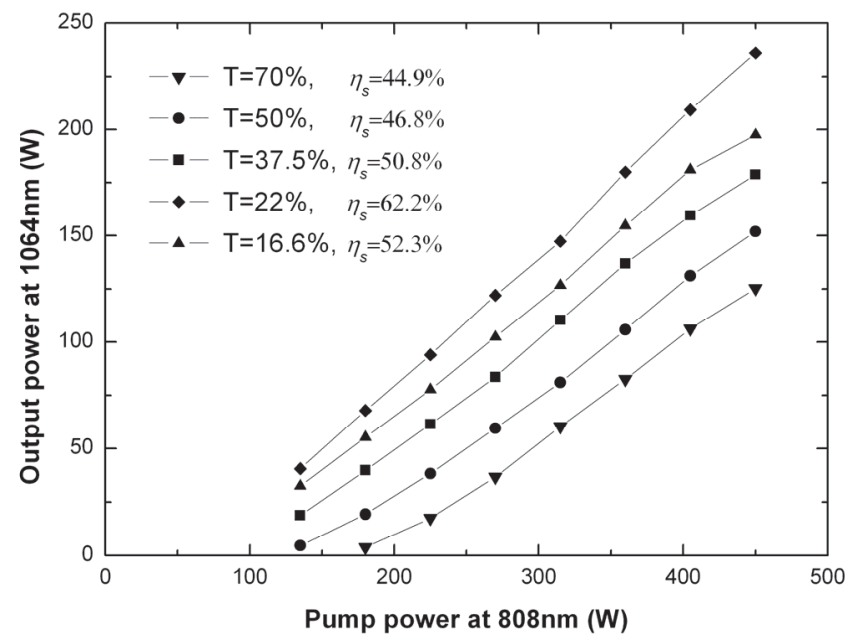

Fig. 7. Output power versus pump power for Nd:YAG ceramics laser with different coupling mirrors.

Referring to the former experimental record of a Nd:YAG single crystal with the same concentration and size using in this system with an output coupling mirror of $\mathrm{T}=70 \%$, we made a comparison between ceramics and crystal, which was shown in Figure. 8. The optical to optical efficiencies were $29 \%$ and $27 \%$ for the ceramics laser and for the single crystal laser, respectively. The corresponding slope efficiency was $46 \%$ for ceramics laser, and $44 \%$ for single crystal laser. It showed that these two kinds of laser materials share extraordinary the same laser output properties in quasi-CW operating.

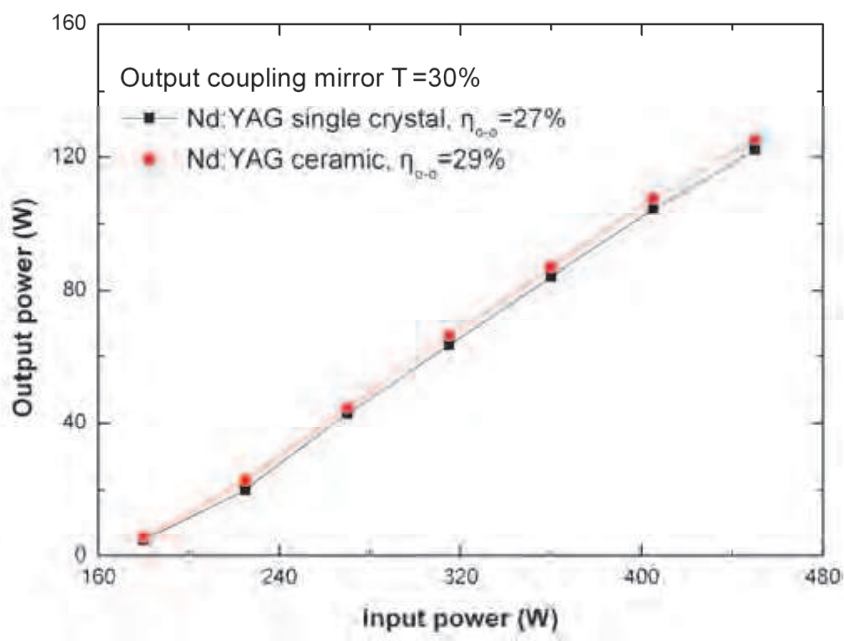

Fig. 8. Comparing output power of Nd:YAG ceramics and Nd:YAG single crystal at the same condition. 
Figure. 9 showed the two and three-dimensional beam profiles of the Nd:YAG ceramics laser from CCD. Some interference stripes could be seen because the cavity length was fixed and the pass length differences between the transmitted beams were multiple numbers of the laser wavelength. It can be eliminated just by adjusting the cavity length slightly. The divergence angle of laser beam was measured about 12 mrad. For high power rod Nd:YAG lasers, thermal lensing and thermal stress-induced birefringence play very important roles. They would result a distortion of the laser beam and cause a significant decrease in beam quality and optical efficiencies. The detailed study will be explored later.

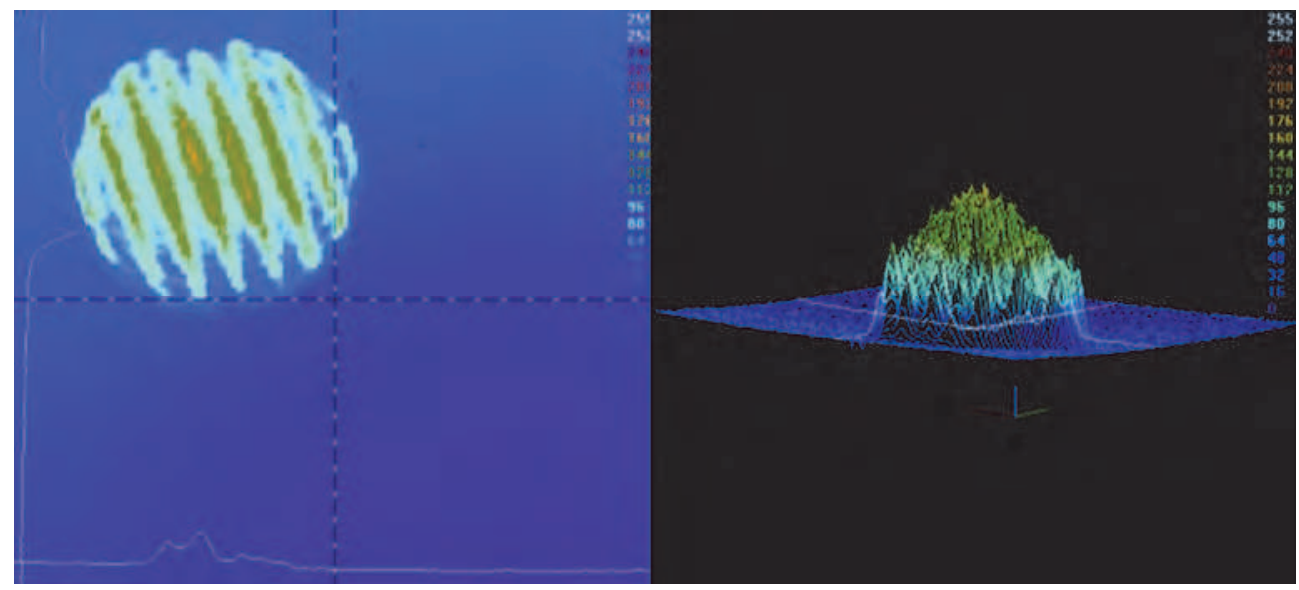

Fig. 9. Two and three-dimensional beam profiles o of a 236W Nd:YAG ceramics laser from CCD.

In conclusion, a high efficiency high power quasi-CW Nd:YAG ceramics rod laser operating at $1064 \mathrm{~nm}$ was demonstrated by using compact quasi-CW LD stacked arrays side-pumping system. High average output power of $236 \mathrm{~W}$ was achieved under $450 \mathrm{~W}$ pumping, corresponding to an optical-to-optical efficiency of $52.5 \%$ and slope-efficiency of $62 \%$.

\subsection{Q-switched Nd:YAG ceramics laser}

Based on previous work, we improved the system and thus demonstrated a high energy electro-optical Q-switched Nd:YAG ceramics laser. With $420 \mathrm{~W}$ quasi-CW LDA pumping at $808 \mathrm{~nm}$ and Q-switched repetition rate at $100 \mathrm{~Hz}, 50 \mathrm{~mJ}$ pulsed laser at $1064 \mathrm{~nm}$ was obtained with pulse width of $10 \mathrm{~ns}$, an average output power of $5 \mathrm{~W}$ and peak power of 5 MW. Its corresponding slope-efficiency was $29.8 \%$.

The experimental setup of LDA side-pumped electro-optical Q-switched Nd:YAG laser was shown schematically in figure. 10. The radiation light emitted from the ceramics rod was first linearly polarized by a polarizer and then introduced a phase difference of a quarter of a wavelength through the quarter-wave plate. A KD*P nonlinear crystal was employed as a Pockels cell Q-switch with longitudinal field. The total length of the cavity was about $260 \mathrm{~mm}$. 


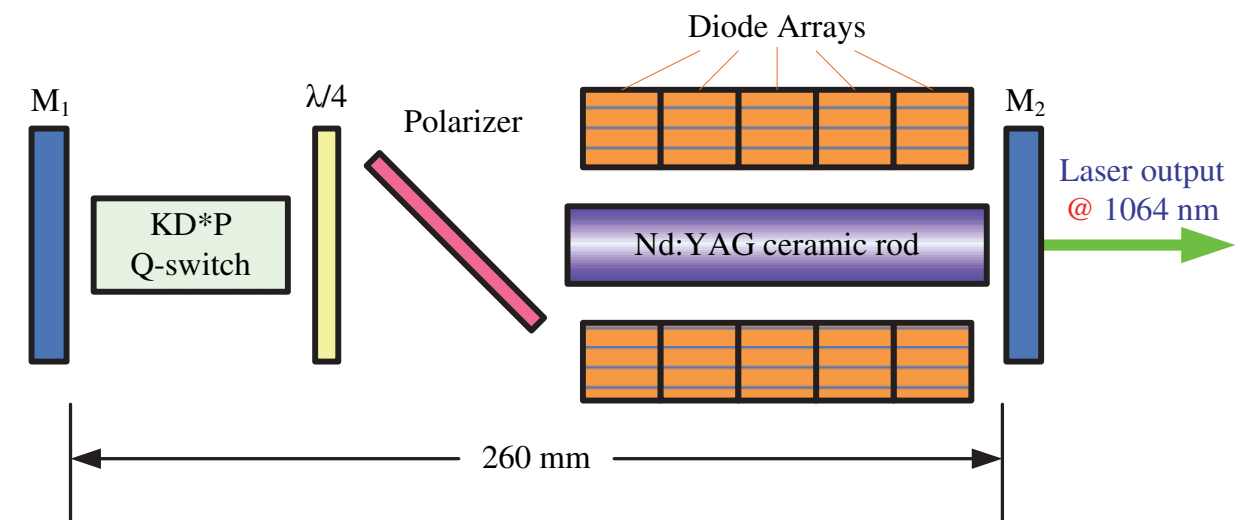

Fig. 10. Schematic diagram of experimental setup for side-pumped E-O switched Nd:YAG ceramics rod laser.

We employed two Nd:YAG samples with the same concentration and size in our experiment. One was ceramics, and the other was single crystal. Figure. 11. showed the comparative laser output power of the two samples with different conditions. At first, the two Nd:YAG lasers were easy to operate at quasi-CW mode without the polarizer, quarterwave plate and KD*P Q-switch. Their average output power and pulse energy increased almost linearly with the increasing of the pumping energy. The corresponding slope efficiency was $46 \%$ for ceramics laser, and $44 \%$ for single crystal laser. And the optical to optical efficiencies were $29 \%$ and $27 \%$ for the ceramics laser and for the single crystal laser, respectively. When those modulating devices were inserted into the laser cavity, the actively

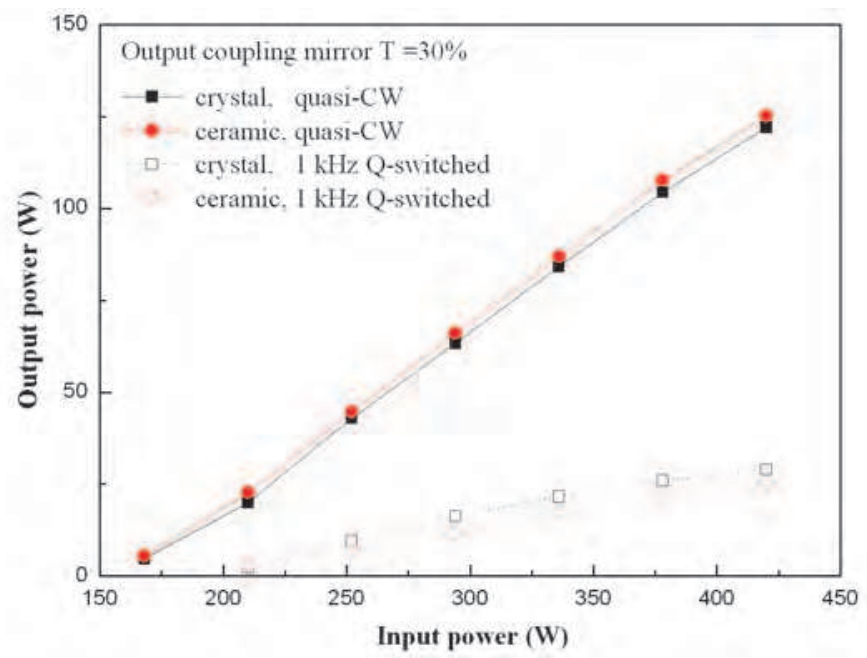

Fig. 11. Comparing output power of Nd:YAG ceramics and single crystal at the same condition. 
Q-switched operation has been observed. Both of the single crystal and ceramics Nd:YAG lasers are affected by the thermal depolarization losses, so caused a little roll over of the E-O Q-switched output power curves and a significant decrease of the optical efficiencies when compared with quasi-CW operations.
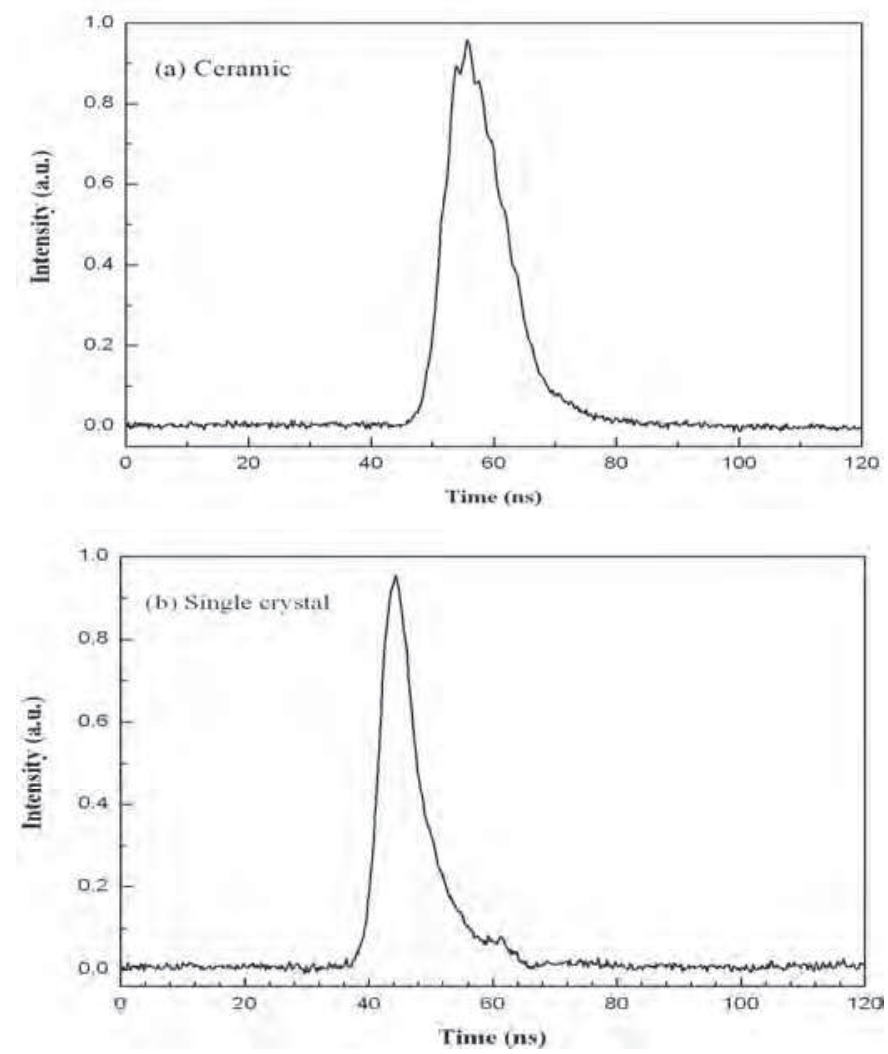

Fig. 12. Single pulse waveform of Electro-Optical Q-switched Nd:YAG lasers under modulating repetition rate of $1 \mathrm{kHz}$. (a) ceramics; (b) single crystal.

Under the max average pumping power of $420 \mathrm{~W}$ and $1 \mathrm{kHz}$ modulating rate, the slope efficiency of ceramics sample was $15.2 \%$ and its pulse width is $12 \mathrm{~ns}$ and those of single crystal sample were $17.5 \%$ and 9.6 ns. Figure. 12. showed the single pulse shape from electro-optical Q-switched Nd:YAG crystal and ceramics lasers. The above data showed that these two kinds of laser material shared very similar laser output characteristics. The ceramics has a little better performance in quasi-CW operating while the single crystal was better in pulse operation. We speculated that the polycrystalline structure inside the ceramics body, which changes the path length of photons in the rod and adds the scattering losses of the cavity, extended the waveform distortion of the Q-switched laser pulse, and resulted in lower efficiency and broaden pulse width. As well as Nd:YAG single crystal, $\mathrm{Nd}$ :YAG ceramics are affected by the thermal effects when high energy pulse operation. The 
detail research on the thermal-optical effects of Nd:YAG ceramics laser is to be explored in another paper.
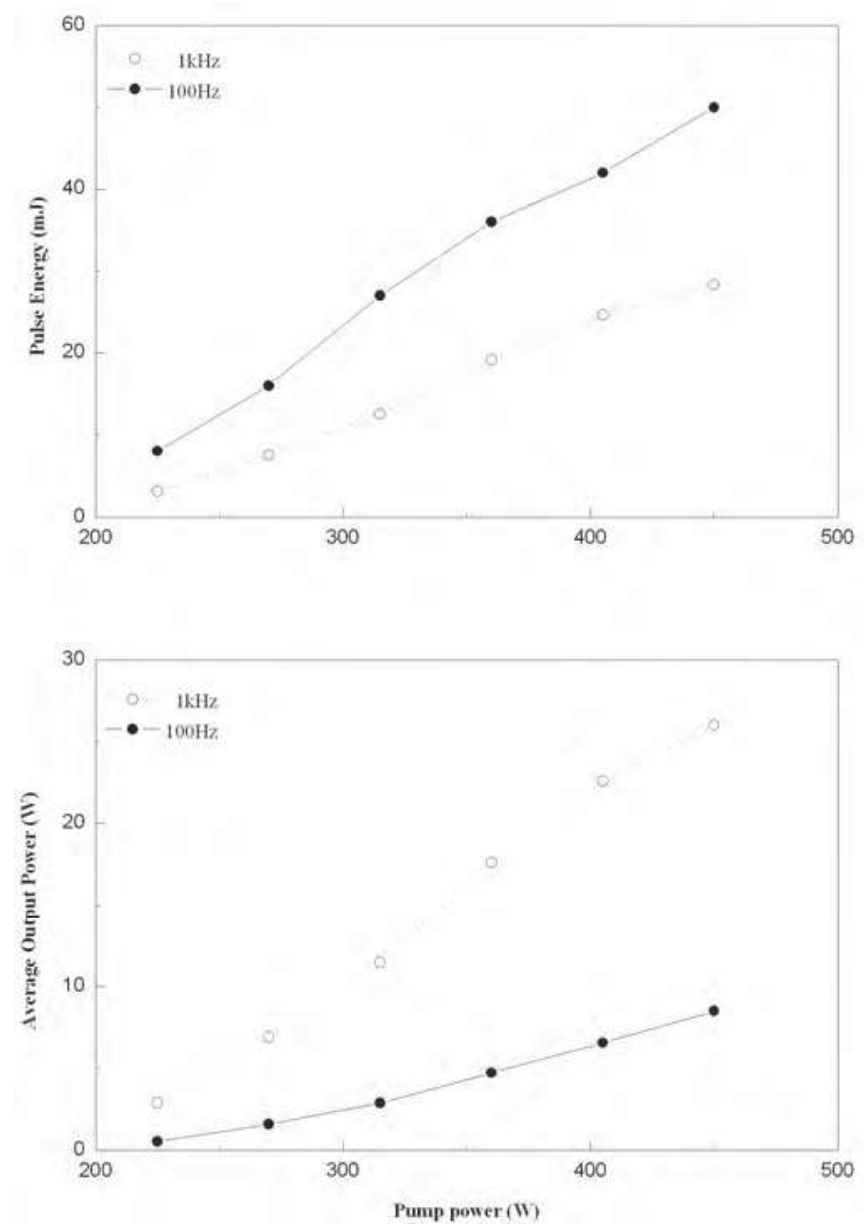

Fig. 13. Paverage power, pulse energy vs. Ppump and repetition rate of Nd:YAG ceramics laser.

Next we changing the pumping condition and modulating rate to $100 \mathrm{~Hz}$ operation, and compared the pulse performances of Nd:YAG ceramics laser under different repetition rates. The average output power and pulse energy as functions of the pumping energy with different repetition rates have been measured and plotted in Figure. 13. With $420 \mathrm{~W}$ max average pumping power, an average output power of $28.3 \mathrm{~W}$ was achieved under the repetition rate of $1 \mathrm{kHz}$. The pulse energy was $28.3 \mathrm{~mJ}$ and its peak power was $2.36 \mathrm{MW}$ with pulse width of 12 ns. Its slope-efficiency was $15.2 \%$. While under the modulating repetition rate of $100 \mathrm{~Hz}$, the average output power of $5 \mathrm{~W}$ with pulse width of $10 \mathrm{~ns}$ was observed. The pulse energy was $50 \mathrm{~mJ}$ and its peak power was $5 \mathrm{MW}$. And the 
corresponding slope-efficiency was $29.8 \%$. Electro-optical Q-switched ceramics laser with higher modulating repetition rates generated higher average output power but broader pulse width and lower pulse energy and peak power. No saturation phenomenon was observed and higher output energy could be in expectation. Because the thermal build up of higher repetition rate pulse laser is more serious than that of lower repetition rate pulse laser, so the thermal depolarization losses of $1 \mathrm{k} \mathrm{Hz}$ pulse laser were higher than those of 100 $\mathrm{Hz}$ pulse laser, which resulted lower efficiency than the latter.

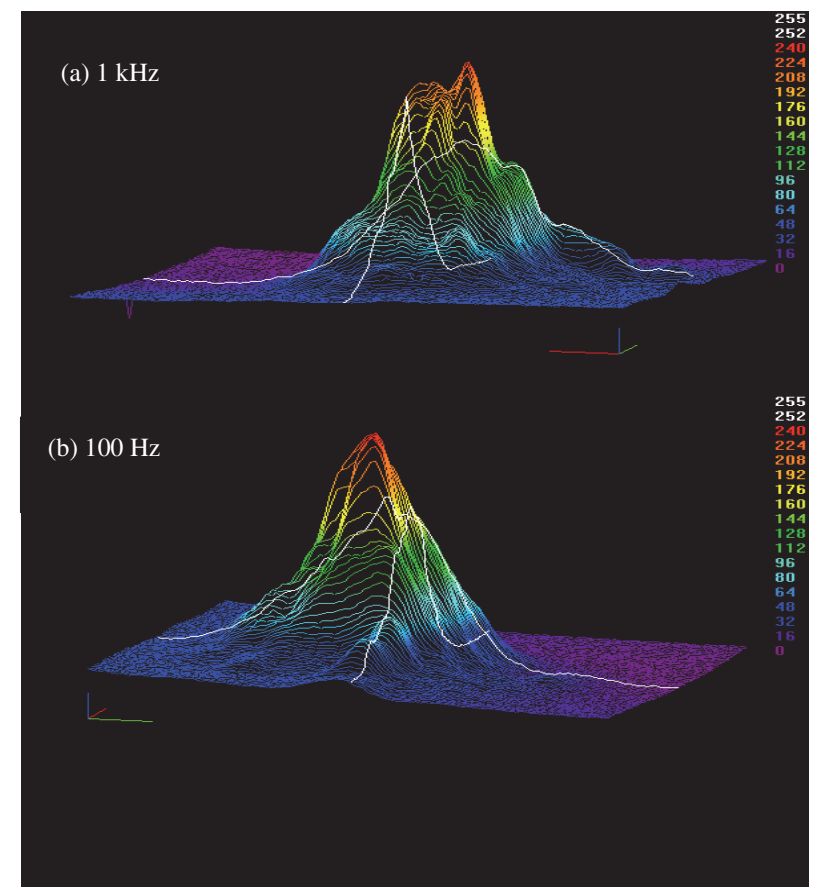

Fig. 14. Beam profile of Nd:YAG ceramics laser under different modulating rates from CCD.

Figure. 14. showed the three-dimensional beam profiles of the pulse Nd:YAG ceramics laser with different modulating rates and under the max pumping power of $420 \mathrm{~W}$ from CCD. They were approximate Gaussian beam intensity distribution, but a little distortion indicated some thermal stress-induced birefringence was existed.

In conclusion, a high energy electro-optical Q-switched Nd:YAG ceramics laser has been demonstrated by employing a quite uniformly compact side-pumping system. The laser parameters between ceramics and single crystal Nd:YAG lasers have been compared and the pulse characteristics of ceramics laser with different repetition rates have been discussed in detail. With $100 \mathrm{~Hz}$ modulating rate, output energy of $50 \mathrm{~mJ}$ has been attained with pulse width of $10 \mathrm{~ns}$ and average output power of $5 \mathrm{~W}$. And its corresponding peak power was 5 MW. While with $1 \mathrm{kHz}$ modulating rate, output energy of $28.3 \mathrm{~mJ}$ has been achieved with pulse width of $12 \mathrm{~ns}$ and an average output power of $28.3 \mathrm{~W}$. Table.1. Summarized the measured laser parameters with the effective pumping energy of $420 \mathrm{~mJ}$ at $1064 \mathrm{~nm}$. It approved in experimental that Nd:YAG ceramics has comparable good performance with 
Nd:YAG single crystal in mJ-level energy laser output. By optimizing the design of the laser cavity, adopting higher pumping power and choosing proper repetition rate, the Nd:YAG ceramics Electro-optical Q-switched laser will obtain better performance with higher pulse energy and narrower line width as well as better beam quality.

\begin{tabular}{|l|l|l|l|l|l|}
\hline 1at.\% Ceramics & $\begin{array}{l}\text { Average output } \\
\text { Power }\end{array}$ & Pulse energy & Pulse width & Peak power & $\begin{array}{l}\text { Slope } \\
\text { efficiency }\end{array}$ \\
\hline Quasi-CW & $236 \mathrm{~W}$ & $236 \mathrm{~mJ}$ & $160 \mu \mathrm{s}$ & $1.6 \mathrm{~kW}$ & $62 \%$ \\
\hline Pulse $(1 \mathrm{kHz})$ & $28.3 \mathrm{~W}$ & $28.3 \mathrm{~mJ}$ & $12 \mathrm{~ns}$ & $2.36 \mathrm{MW}$ & $15.2 \%$ \\
\hline Pulse $(100 \mathrm{~Hz})$ & $5 \mathrm{~W}$ & $50 \mathrm{~mJ}$ & $10 \mathrm{~ns}$ & $5 \mathrm{MW}$ & $29.8 \%$ \\
\hline
\end{tabular}

Table 1. Experimental laser parameters of Nd:YAG lasers at $1064 \mathrm{~nm}$ with effective pumping energy of $390 \mathrm{~W}$.

\section{Q-switched pulsed ceramics laser}

\subsection{CW and Q-Switched performance of a Yb:YAG/YAG composite thin disk ceramics laser}

Since the emergence of semiconductor laser diodes (LD) that emit at $900 \sim 1100 \mathrm{~nm}$, high power LD array are used as stabilized pumping source. The $\mathrm{Yb}$ doped laser material with the pumping wavelength requirement at this wavelength range attracts a lot of attention. [17] Figure. 15. shows the energy level of $\mathrm{Yb}^{3+}$ ion in the crystal $\mathrm{Yb}$ :YAG.[18] $\mathrm{Yb}^{3+}$ ion has very simple energy diagram with ${ }^{2} \mathrm{~F}_{7 / 2}$ as lower level and ${ }^{2} \mathrm{~F}_{5 / 2}$ as excited state manifolds separated by about $10,000 \mathrm{~cm}^{-1}$. The laser wavelength of $\sim 1030 \mathrm{~nm}$ with transition of ${ }^{2} \mathrm{~F}_{5 / 2}$ ${ }^{2} \mathrm{~F}_{7 / 2}$ has a terminal level of $612 \mathrm{~cm}^{-1}$ above the ground states. While the thermal energy at room temperature is $200 \mathrm{~cm}^{-1}$, the terminal state is thermally populated making the $\mathrm{Yb}: \mathrm{YAG}$ a quasi-three level system. At room temperature, the thermal population of the lower laser level is about $5.5 \%$.
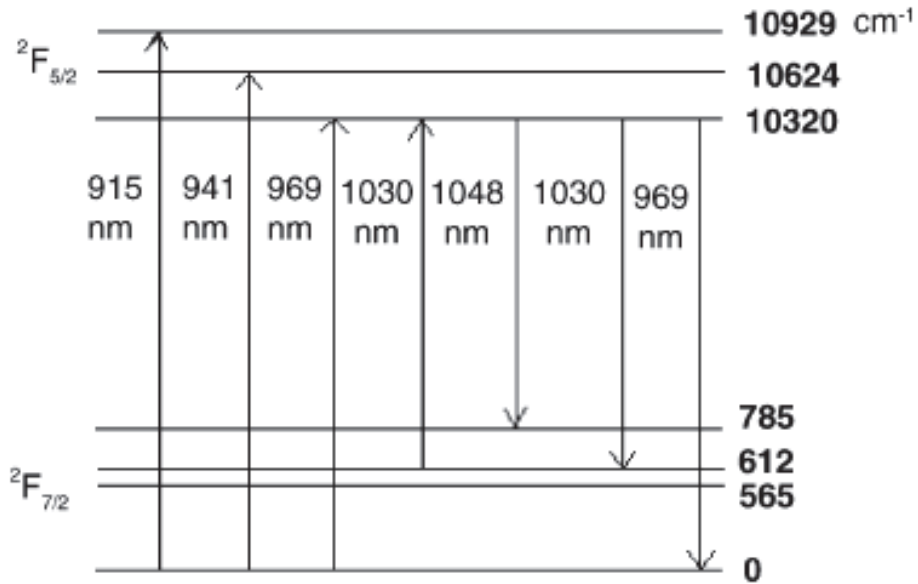

Fig. 15. Energy level of $\mathrm{Yb}$ [2] 
Table 2 summarized physical, chemical and laser properties of Yb:YAG single crystal. [1921] Comparing to the $\mathrm{Nd}: \mathrm{YAG}$ laser material, $\mathrm{Yb}$ doped laser material have the merits of (1) wide pumping range, (2) high quantum efficiency of over $90 \%$, (3) longer upper-state lifetime of $\sim 1 \mathrm{~ms}$, (4) no excited state absorption, (5) no up-conversion, and (6) minimal concentration quenching. With the fast development of the Nd:YAG transparent ceramics, the $\mathrm{Yb}$ doped laser ceramics also shows its potential as one of the ideal candidates for highpower laser application.

\begin{tabular}{|c|c|}
\hline Crystal Structure & Cubic \\
\hline Lattice Parameters (nm) & 1.201 \\
\hline Melting Point (K) & 2243 \\
\hline Moh Hardness & 8.5 \\
\hline Density $\left(\mathrm{g} / \mathrm{cm}^{3}\right)$ & $4.56 \pm 0.04$ \\
\hline Specific Heat $(0-20)\left(\mathrm{J} / \mathrm{g} . \mathrm{cm}^{3}\right)$ & 0.59 \\
\hline Modulus of Elasticity (GPa) & 310 \\
\hline Young's Modulus $\left(\mathrm{Kg} / \mathrm{mm}^{2}\right)$ & $3.17 * 104$ \\
\hline Poisson Ratio (est.) & 0.3 \\
\hline Tensile Strength (GPa) & $0.13 \sim 0.26$ \\
\hline \multicolumn{2}{|l|}{ Thermal Expansion Coefficient $(/ \mathrm{K})\left(0 \sim 250^{\circ} \mathrm{C}\right)$} \\
\hline [100]Direction & $8.2 * 10^{-6}$ \\
\hline [110]Direction & $7.7^{*} 10^{-6}$ \\
\hline [111]Direction & $7.8 * 10^{-6}$ \\
\hline Thermal Conductivity $(\mathrm{W} / \mathrm{m} / \mathrm{K})$ & $14 @ 20^{\circ} \mathrm{C}, 10.5 @ 100^{\circ} \mathrm{C}$ \\
\hline Thermal Optical Coefficient (dn/dT, /K) & $7.3^{*} 10-6$ \\
\hline Thermal Shock Resistance $(\mathrm{W} / \mathrm{m})$ & 790 \\
\hline Laser Transition & ${ }^{2} \mathrm{~F}_{5 / 2} \rightarrow{ }^{2} \mathrm{~F}_{7 / 2}$ \\
\hline Laser Wavelength & $1030 \mathrm{~nm}, 1048 \mathrm{~nm}$ \\
\hline Photon Energy (J) & 1.93*10-19 @ 1030nm \\
\hline Emission Linewidth (nm) & 9 \\
\hline Emission Cross Section $\left(\mathrm{cm}^{2}\right)$ & $2.0 * 10-20$ \\
\hline Fluorescence Lifetime (ms) & 1.2 \\
\hline Diode Pump Band (nm) & 940 or 970 \\
\hline Pump Absorption Band Width (nm) & 8 \\
\hline Index of Refraction & 1.82 \\
\hline Thermal Optical Coefficient (/K) & $9 * 10^{-6}$ \\
\hline Loss Coefficient $\left(\mathrm{cm}^{-1}\right)$ & 0.003 \\
\hline
\end{tabular}

Table 2. Physical and chemical property of Yb:YAG

Comparing to rod shape medium in which heat is along the radius of the rod, so there is strong thermal gradient induced lensing and birefringence, in the thin disk shaped gain medium heat is extracted through the large faces with thermal gradients which is established across the smallest dimension and aligned with the beam propagation direction. [22] But thermo-mechanical distortion is still the bottleneck of high-power thin disk laser. Researchers brought up the idea of using composite media. During the pumping process, the undoped part of the medium helps to defuse the heat generated by the doped part, 
because the thermal conductivity of undoped part is usually higher than that of doped part. In the case of Yb:YAG/YAG medium, the undoped YAG acts as a passive heat sink and rebuilds the temperature field, especially along the thickness direction, and it seems that there is an imaginary cooling effect on the front face of the gain medium. [23] So by using a composite gain medium, which consists of both Yb:YAG and undoped YAG, the bending of the medium can be eliminated to some degree. Additionally, the composite medium eliminates the radiation trapping to a larger degree because the undoped YAG mitigates the effects of total internal reflection at the undoped-YAG-air interface. [24]

Figure. 16. shows the pictures of the composite Yb:YAG/YAG thin disk ceramics made by BAIKOWSKI, Japan. The thin disk is $\sim 10 \mathrm{~mm}$ in diameter with very thin absorbing part of the disk $(\sim 0.6 \mathrm{~mm})$ bonded together with a thicker undoped piece of YAG ceramics $(\sim 2.5$ $\mathrm{mm})$. The doping concentration is 9.8 at. \% in the doped part. The composite ceramics disk is AR coated for the wavelength of $\sim 930-970 \mathrm{~nm}$ and laser radiation $1030 \mathrm{~nm}$ at the front side and HR coated for both wavelengths at the back side. Figure.17. shows the double-pass absorptivity of the disk ceramics. There are mainly three absorption peaks in the range of $900 \mathrm{~nm} \sim 1100 \mathrm{~nm}: 937 \mathrm{~nm}, 968 \mathrm{~nm}$ and 1027nm, with absorption efficiency of $\sim 75 \%, 58 \%$ and $38.7 \%$, absorption bandwidth of $\sim 37 \mathrm{~nm}, 10 \mathrm{~nm}$ and $14 \mathrm{~nm}$ respectively.
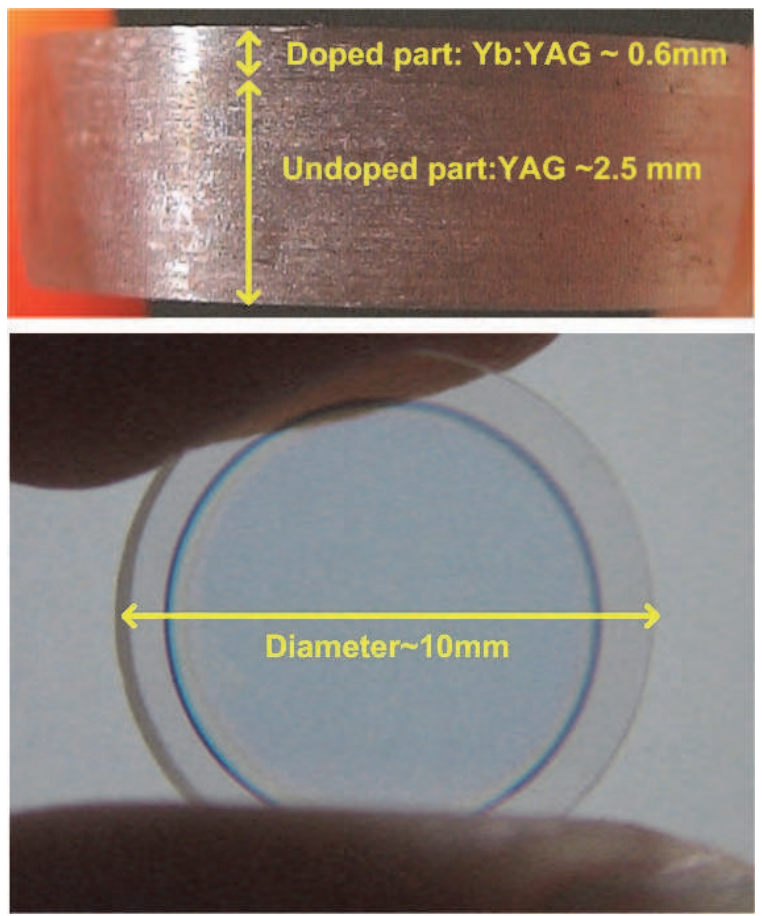

Fig. 16. Pictures of Yb:YAG/YAG composite transparent ceramics disk 


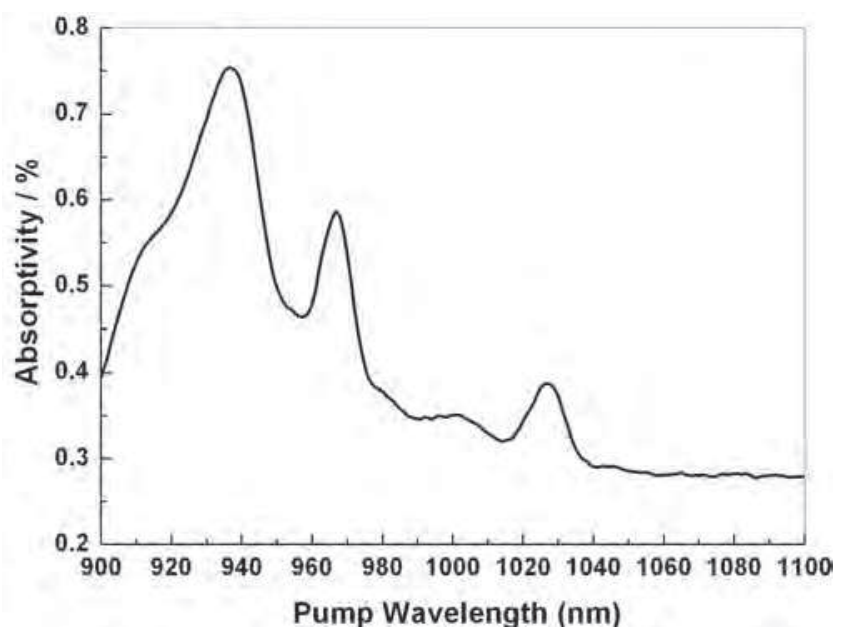

Fig. 17. Double-pass absorptivity of the composite ceramics

In order to lengthen the effective absorbing length in the thin-disk medium and make a good overlap between pump and resonator mode, a face-pumped CAMIL structure is chosen. With this structure, diode pump radiation is injected into the back face of the disk and then reflected by the face several times. The schematic diagram of the experimental setup is shown in 18. The laser medium is a composite Yb:YAG/YAG thin disk ceramics as described above. It is fixed with a layer of indium onto a heat sink, which is cooled with water from the back side. A collimated LD array with central wavelength at $970 \mathrm{~nm}$ working at $15{ }^{\circ} \mathrm{C}$ is used as pump source. By a focal length of $\sim 9.4 \mathrm{~cm}$ lens, the pumping light is focused on the back side of the ceramics and the unabsorbed pumped radiation is reflected for another turn of absorption, i.e., the effective absorbing length is twice the length of the doped ceramics. A dichroic beam splitter $\left(45^{\circ}\right)$ which is coated with AR film at $970 \mathrm{~nm}$ and HR film at 1030nm is inserted between the focusing lens and the composite ceramics for redirecting the laser to the output couplers.

In the CW mode, output couplers with the same radius of curvature of $100 \mathrm{~mm}$, and transmissions of $1 \%, 2 \%, 5 \%$ and $10 \%$ are used respectively. The whole cavity length is $\sim 80$ $\mathrm{mm}$. In the Q-switched mode, output coupler with transmission of $10 \%$ is used. The output laser power is measured by a power meter (OPHIR, NOVA II) and the spectrum is recorded by a spectroscopy (YOKOGAWA, AQ6370), while the pulse width is recorded by an oscillograph (Lecroy, WR62XR).

In the CW mode, the laser output power increases as the pump power increases with different output couplers, as shown in figure. 19. Up to $1.05 \mathrm{~W} \mathrm{CW}$ power is achieved with optical to optical efficiency of $5.25 \%$ with $2 \%$ output coupler. Central laser wavelength is at $1031 \mathrm{~nm}$, as shown in Fig.20. We also get Q-switched output of the laser using an acoustooptic (A-O) Q-switch. We insert the A-O Q-switch device (Gooch \& Housego, M080-2G) into the cavity with $10 \%$ transmission output coupler. Stable operation is achieved with the repetition rate of $1 \mathrm{kHz}, 5 \mathrm{kHz}, 10 \mathrm{kHz}, 20 \mathrm{kHz}$ and $30 \mathrm{kHz}$, along with the average output power of $0.44 \mathrm{~W}, 0.446 \mathrm{~W}, 0.452 \mathrm{~W}, 0.461 \mathrm{~W}$ and $0.47 \mathrm{~W}$ respectively. Figure. 21. shows the width of the pulse enlarges with the increasing repetition rate. Figure 22 shows the pulse waveform at $1 \mathrm{kHz}$ : a minimal pulse width of $166 \mathrm{~ns}$ and corresponding peak power of 2.6 
kW. Figure. 22. inset also shows the pulse serial, which appears to be a bit unstable but acceptable.

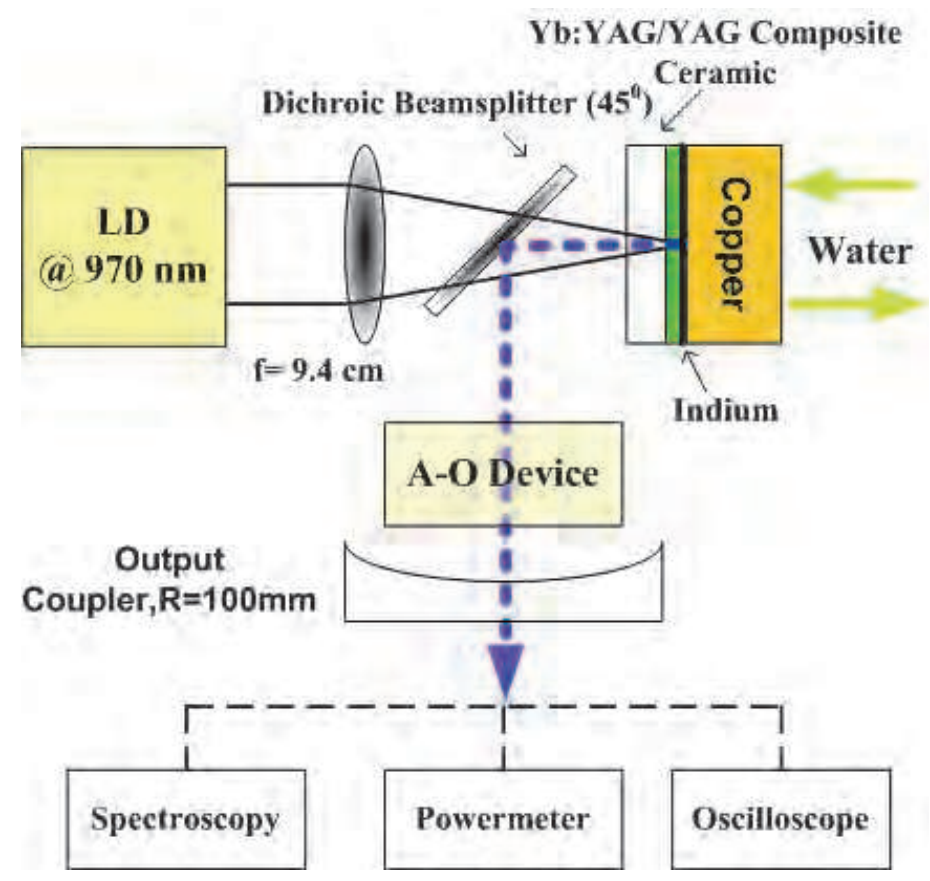

Fig. 18. Schematic diagram of the experimental setup

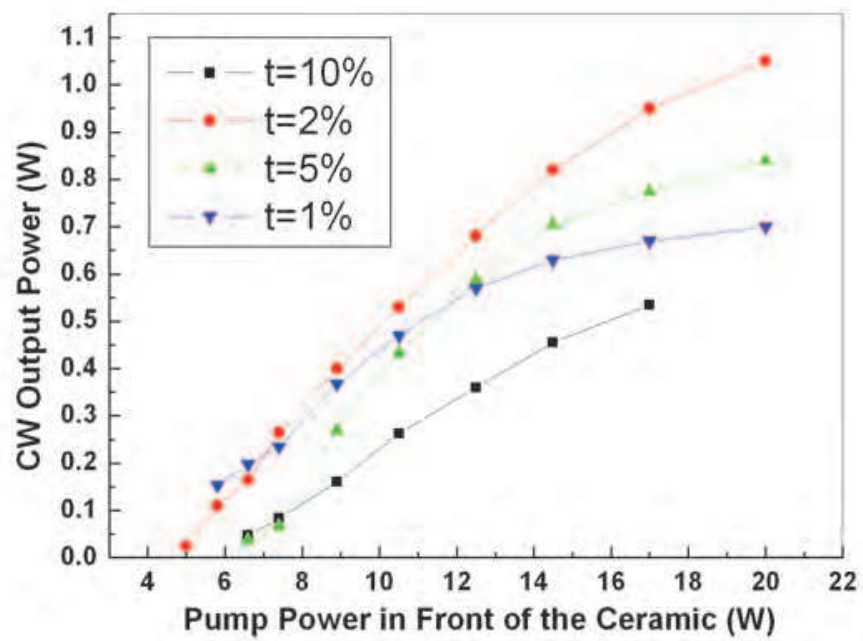

Fig. 19. CW laser output power vs. Pump power with different output transmission 


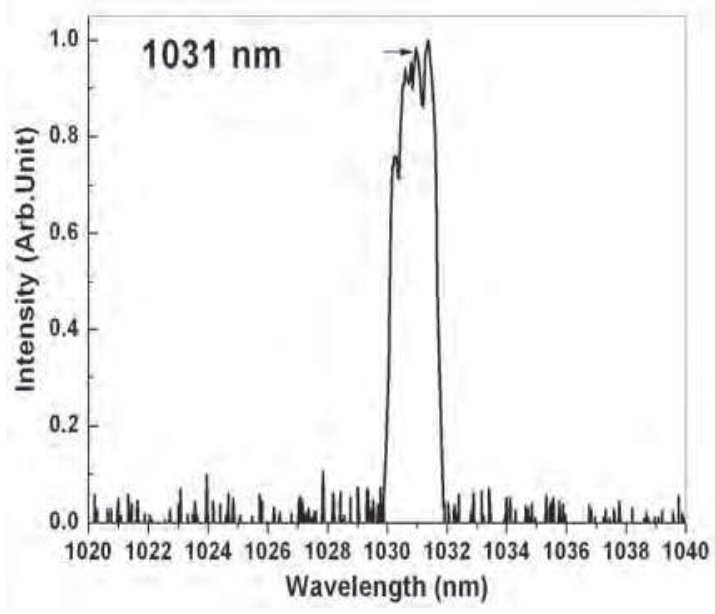

Fig. 20. Laser output spectrum

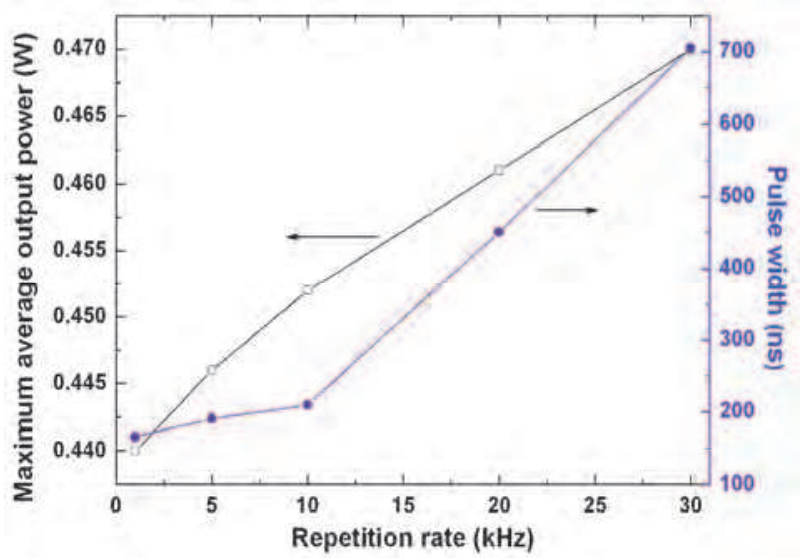

Fig. 21. Average output power and Pulse width vs. Repetition rate

Both for CW and $\mathrm{AO}$ Q-switched mode, the optical to optical efficiency is low according to the data figure 19 and figure 21. But when we considered the actual absorbed pump power, the case would be different. There is only $53.5 \%$ of the pump power can be absorbed at the pump wavelength $970 \mathrm{~nm}$, as shown in Fig.17. Moreover, further measurement reveals that the pumping wavelength drifted dramatically along with the increasing pump power. Figure 23 shows the measured relationship of pump wavelength and pump power while maintaining the temperature of the cooling water at $15^{\circ} \mathrm{C}$. The pumping central wavelength drifts from $970 \mathrm{~nm}$ to $979 \mathrm{~nm}$ with the decreased pumping absorptivity from $53.5 \%$ to $38 \%$, respectively. Figure. 19. also suggests that in higher pump power region, the laser power tended to be "saturated", which is possibly caused by the decreasing absorption efficiency of the medium. Form this experiment, we found that it's difficult to control the pump 
wavelength only by cooling in this pump source. Figure 17 indicates that the lengthened absorbing length inside the laser medium brought about $28 \%$ background absorption of the pump power, which might be caused by the quality of the media. It would raise the laser threshold. Figure 17 also shows that there is another absorption peak at around $1031 \mathrm{~nm}$ where is exactly the output laser wavelength located, indicating the reabsorption effect at $1031 \mathrm{~nm}$. Thus, the increasing pumping power would lead to a stronger reabsorption results in a quick saturation at this wavelength. Moreover, the unabsorbed pump energy would contribute to the difficulty of the population inversion, thermal lensing, which would further reduce the efficiency and the laser output power.

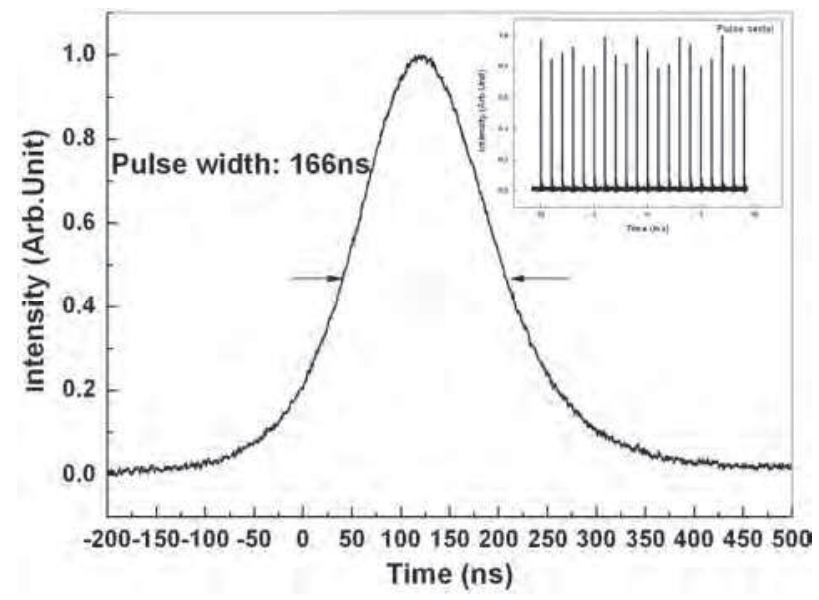

Fig. 22. Pulse profile of minimum pulse width at $1 \mathrm{kHz}$. Inset shows the pulse serial.

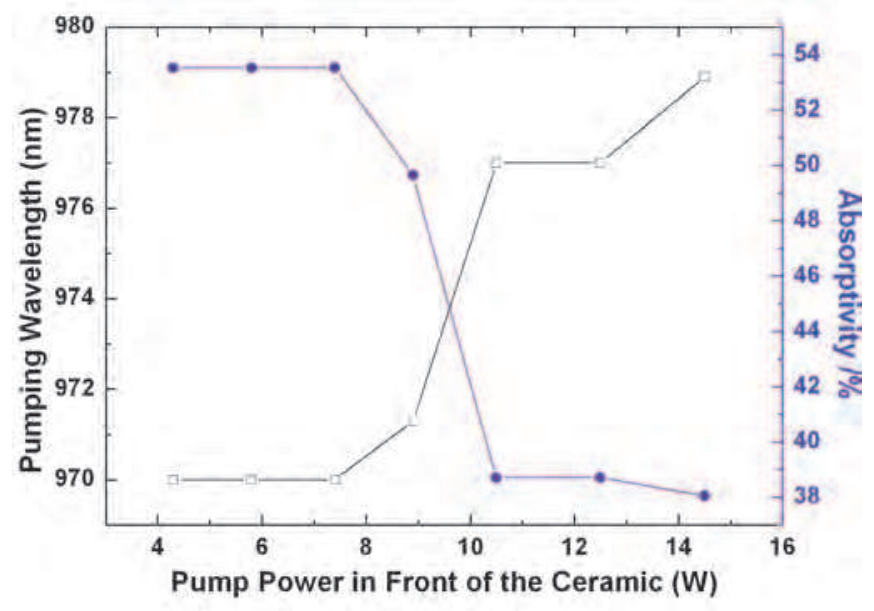

Fig. 23. Pump wavelength drifted with pump power and their corresponding absorptivity 


\subsection{Experimental setup for $933 \mathrm{~nm}$ pumping experiment}

We also study the CW and AO Q-switched laser performance of this Yb:YAG/YAG composite ceramics disk under the pumping wavelength of $\sim 933 \mathrm{~nm}$ in order to further explore the high-power potential of this material by increasing the media absorption of pumping power.

Figure 24 shows the schematic diagram of the experimental setup using $933 \mathrm{~nm}$ pump source. The experimental setup is similar to that of $\sim 970 \mathrm{~nm}$ pump source. The pump source is a fiber coupled LD array with central wavelength at $933 \mathrm{~nm}$ working at $20^{\circ} \mathrm{C}$.

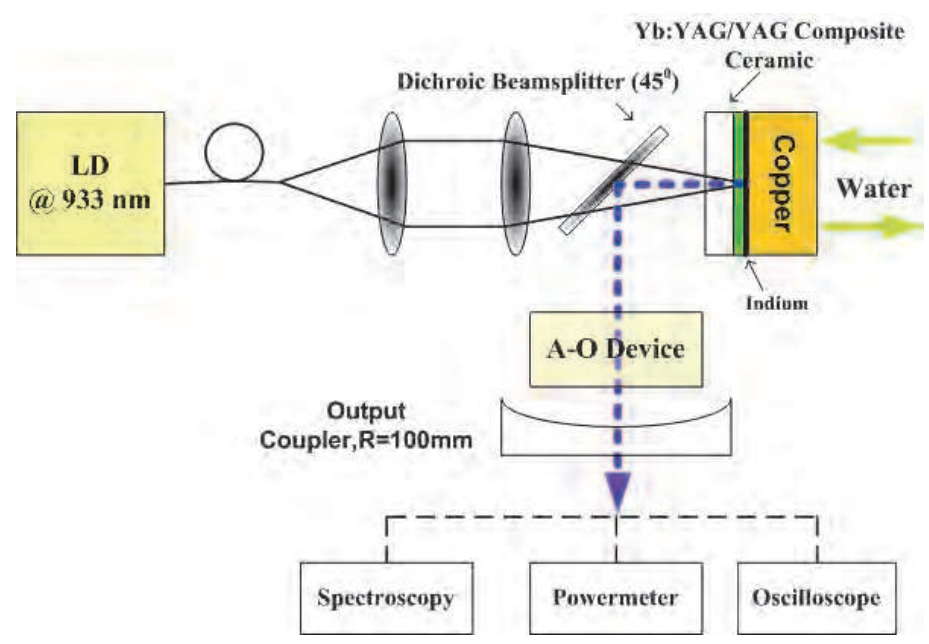

Fig. 24. Schematic diagram of the experimental setup

In the CW mode, output couplers with the same radius of curvature of $100 \mathrm{~mm}$, and transmissions of $1 \%, 2 \%, 5 \%$ and $10 \%$ are used respectively. The whole cavity length is $\sim 80$ $\mathrm{mm}$. In the Q-switched mode, output coupler with transmission of $10 \%$ is used. The output laser power is measured by a power meter (OPHIR, NOVA II) and the spectrum is recorded by a spectroscopy (YOKOGAWA, AQ6370), while the pulse width is recorded by an oscillograph (Lecroy, WR62XR).

In the CW mode, the laser output power increases as the pump power increase with different output couplers, as shown in Figure. 25. When the transmission of the output coupler is $2 \%$, up to $2.575 \mathrm{~W} \mathrm{CW}$ power is achieved with optical-optical efficiency of $17.6 \%$ and slope efficiency of $31.2 \%$. Central laser wavelength is at $1030.2 \mathrm{~nm}$, as shown in Fig.26. Because of the limited output power of the pump source, the maximum output laser power is not high enough. But from figure 25, the output laser shows no saturated intention, which means higher laser output can be achieved in the future.

We also get Q-switched output of the laser using an acousto-optic (A-O) Q-switch. We insert the A-O Q-switch device (Gooch \& Housego, M080-2G) into the cavity with 10\% transmission output coupler. Stable operation is achieved with the repetition rate of $1.1 \mathrm{kHz}$, $5 \mathrm{kHz}, 10 \mathrm{kHz}, 20 \mathrm{kHz}, 30 \mathrm{kHz}$, and $40 \mathrm{kHz}$ along with the average output power of $1.29 \mathrm{~W}$, $2.119 \mathrm{~W}, 2.221 \mathrm{~W}, 2.237 \mathrm{~W}, 2.246 \mathrm{~W}$ and $2.249 \mathrm{~W}$ respectively. Figure 27 shows the width of the pulse enlarges and the maximum peak power of the pulse decreases with the increasing 
repetition rate. Fig. . shows the pulse waveform at $1.1 \mathrm{kHz}$ : a minimal pulse width of $29 \mathrm{~ns}$ and corresponding peak power of $40.4 \mathrm{~kW}$, single pulse energy of $1.17 \mathrm{~mJ}$.

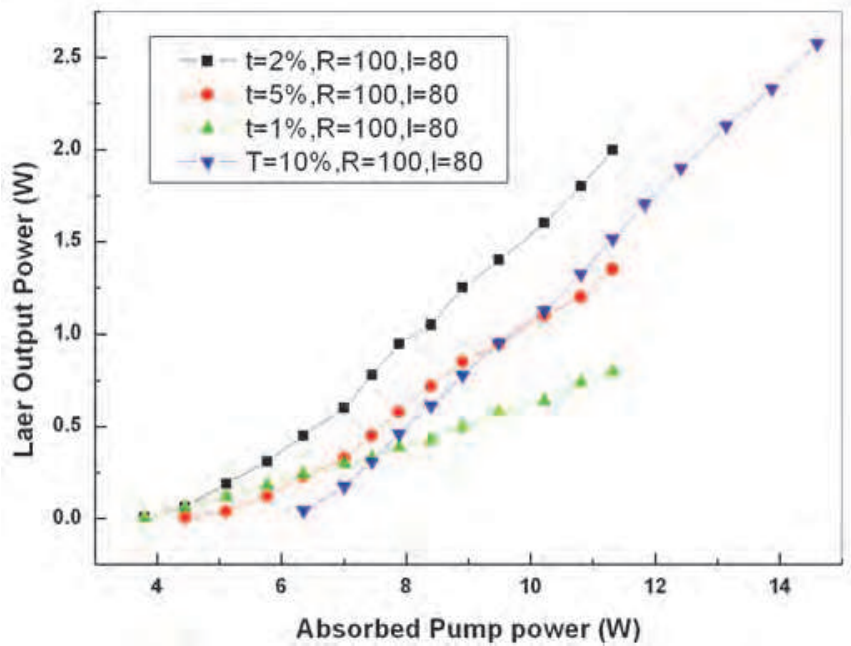

Fig. 25. CW laser output power vs. Pump power with different output transmission

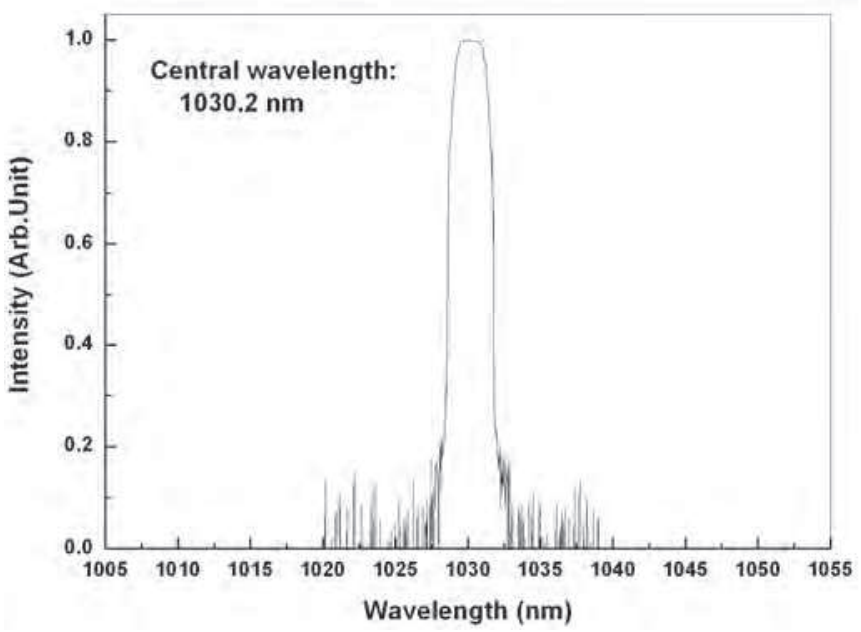

Fig. 26. Laser spectrum 


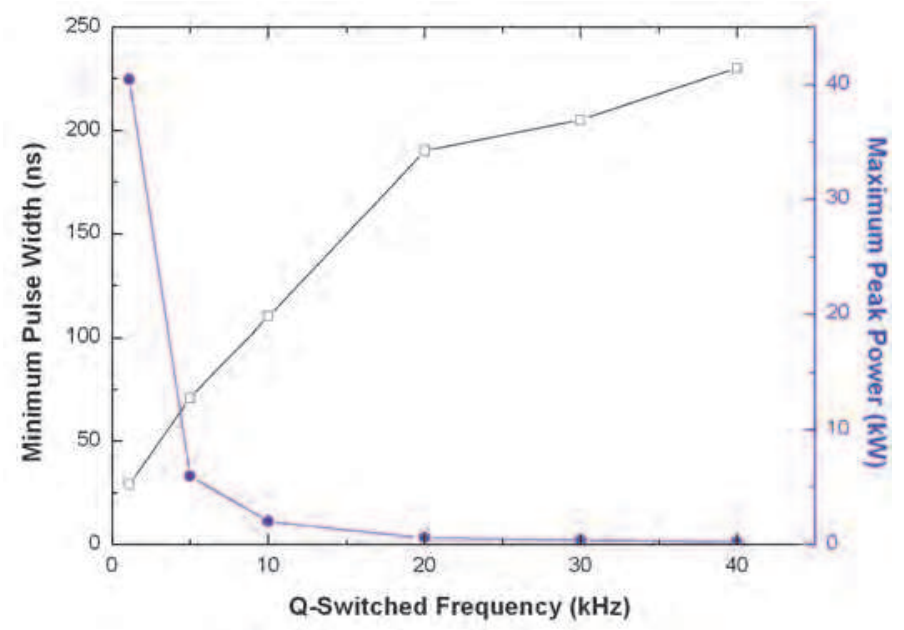

Fig. 27. Maximum peak power and Pulse width vs. Repetition rate

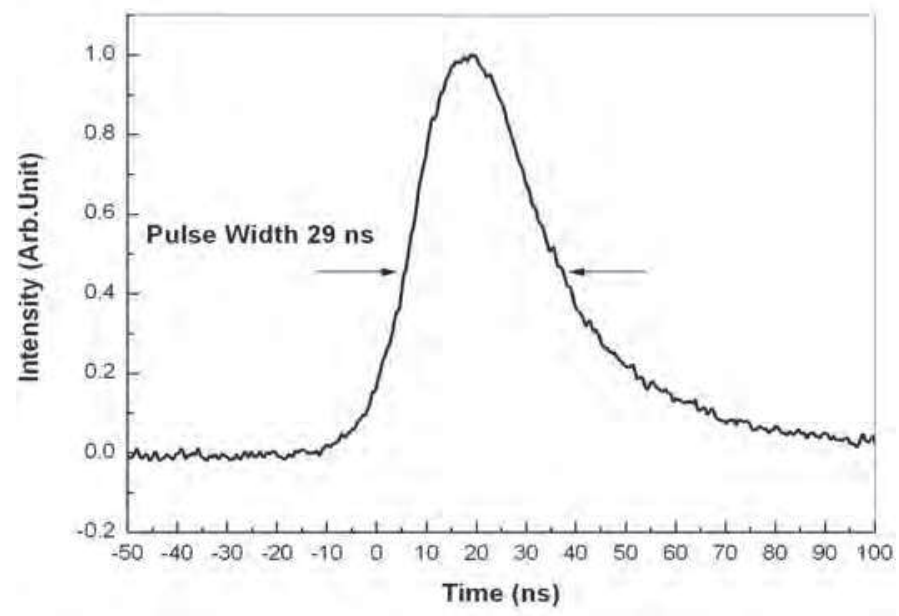

Fig. 28. Pulse profile of minimum pulse width at $1.1 \mathrm{kHz}$.

We demonstrated a CW and Q-switched laser with composite Yb:YAG/YAG ceramics pumped by $970 \mathrm{~nm}$ and $933 \mathrm{~nm}$ LD. For the $970 \mathrm{~nm}$ pumping experiment, a maximum laser power of $1.05 \mathrm{~W}$ with central wavelength at $1031 \mathrm{~nm}$ is obtained. A minimal pulse-width of 166ns and the maximal peak power of $2.6 \mathrm{KW}$ at $1 \mathrm{kHz}$ are achieved, corresponding to an average output power of $0.44 \mathrm{~W}$. The repetition ranged from $1 \mathrm{kHz}$ to $30 \mathrm{kHz}$. For the 933 $\mathrm{nm}$ pumping experiment, a maximum laser power of $2.575 \mathrm{~W}$ with central wavelength at $1030.2 \mathrm{~nm}$ is obtained. A minimal pulse-width of $29 \mathrm{~ns}$ and the maximal peak power of 40.4 $\mathrm{KW}$ at $1.1 \mathrm{kHz}$ are achieved. The repetition ranged from $1.1 \mathrm{kHz}$ to $40 \mathrm{kHz}$. Table 3 summarizes the detailed results. 


\begin{tabular}{|l|l|l|}
\hline Pump source & $\mathbf{9 7 0} \mathbf{~ m m}$ & $\mathbf{9 3 3} \mathbf{~ m}$ \\
\hline Ceramics absorptivity of pump power & $53.5 \% @ 970 \mathrm{~nm}$ & $73 \% @ 933 \mathrm{~nm}$ \\
\hline $\begin{array}{l}\text { Diameter of footprint of focusing } \\
\text { pump laser }\end{array}$ & $500 \mu \mathrm{m}$ & $600 \mu \mathrm{m}$ \\
\hline Central wavelength of laser output & $1031 \mathrm{~nm}$ & $1030.2 \mathrm{~nm}$ \\
\hline FWHM of output laser & $2 \mathrm{~nm}$ & $5 \mathrm{~nm}$ \\
\hline CW maximum output power & $1.05 \mathrm{~W}$ & $2.575 \mathrm{~W}$ \\
\hline CW optical-optical efficiency & $5.25 \%$ & $17.6 \%$ \\
\hline CW slope efficiency & $7 \%$ & $31.2 \%$ \\
\hline Q-switched repetition rate & $1 \mathrm{kHz}-30 \mathrm{kHz}$ & $1.1 \mathrm{kHz}-40 \mathrm{kHz}$ \\
\hline $\begin{array}{l}\text { Maximum average power under Q- } \\
\text { switched mode }\end{array}$ & $0.47 \mathrm{~W}$ & $2.249 \mathrm{~W}$ \\
\hline Shortest pulse-width & $166 \mathrm{~ns}$ & $29 \mathrm{~ns}$ \\
\hline Maximum peak power & $2.6 \mathrm{~kW}$ & $40.4 \mathrm{~kW}$ \\
\hline
\end{tabular}

Table 3. Experimental results of $970 \mathrm{~nm}$ and $933 \mathrm{~nm}$ pumping experiment

\section{6. $\mathrm{Nd}: \mathrm{Y}_{3} \mathrm{Sc}_{1.5} \mathrm{Al}_{3.5} \mathrm{O}_{1}$ ceramics as the disk laser media, dual - wavelength competition output}

The Nd:YAG ceramics has proven its advanced merits $[25,26]$ and can be manufactured commercially. But scientists also exploree forward to develop new kind of ceramics in order to overcome the disadvantage exists in Nd:YAG single crystal. Recently, a new kind of laser material based on Nd:YAG-Nd:YSAG has been prepared, in which $\mathrm{Sc}^{3+}$ replaces $\mathrm{Al}^{3+}$ in YAG. Because $\mathrm{Sc}^{3+}$ has a larger size than $\mathrm{Al}^{3+}$, the entrance of $\mathrm{Sc}^{3+}$ leads to lattice expansion. In this way, more $\mathrm{Nd}^{3+}$ can be accommodated in the lattice, in other words, higher doping level is expected comparing to Nd:YAG. By far, highly and homogeneous doped Nd:YSAG has been fabricated successfully. Due to the associated increase of the absorption coefficient, it's possible for us to use thinner laser media, which promises a higher cooling efficiency because of the higher surface area per unit volume. Thus we can reduce optical distortion and thermal stress, which are important for improving the laser beam quality. Moreover, the enhanced emission intensity, prolonged fluorescence lifetime [27, 28 ] and lower threshold comparing to the quasi-four-level $\mathrm{Yb}^{3+}$ doped material together highlight this novel material. It's a suitable media for short-pulse microchip laser. 10ps ultra short-pulse laser has been generated from a passive mode-locked Nd:YSAG ceramics laser [29]. Therefore, transparent ceramics Nd:YSAG will find its way to the application of thin-disk laser and high-power miniature laser.

But Nd:YSAG ceramics is an interesting material besides its merits discussed above. In our experiment, we demonstrated a dual-wavelength competitive laser output in $\mathrm{Nd}: \mathrm{Y}_{3} \mathrm{Sc}_{1.5} \mathrm{Al}_{3.5} \mathrm{O}_{12}$ ceramics disk. In former published papers, many scientists have reported dual-wavelength in many laser materials, such as J. Lu et al. [30]. Yoichi Sato et al. also reported the appearance of dual-wavelength in Nd:YSAG [29]. We further compare the laser spectra in Nd:YAG and Nd:YSAG and then figures out possible reasons for this interesting 
phenomenon from the view of the material structure. The competitiveve behavior of these two wavelengths prognosticates a possible simpler way to generate Terahertz radiation.

Splitting laser level of Nd3+ in YSAG and Fluorescence spectra of Nd:YSAG ceramics Since introducing Sc3+ into the Nd:YAG, which means that the surrounding of the optical center $\mathrm{Nd} 3+$ is modified, the energy level structure would be altered. By using a model Hamiltonian that assumes D2 site symmetry for the $\mathrm{Nd} 3+$ ions in the garnet lattice, John B. Gruber et al. figured out the energy level of Nd:YSAG [31]. The splitting laser levels of Nd3+ in $\mathrm{YSAG}^{-4} \mathrm{~F}_{3 / 2}$ and ${ }^{4} \mathrm{I}_{11 / 2}$-are shown in Figure. 29.

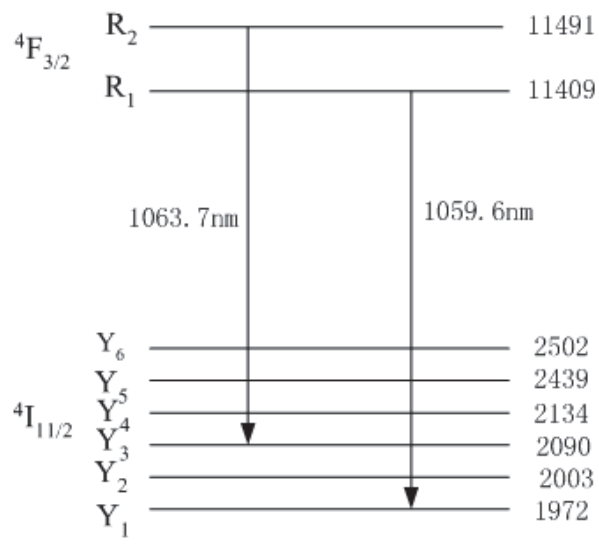

Fig. 29. The splitting laser level of $\mathrm{Nd}^{3+}$ in $\mathrm{YSAG},{ }^{4} \mathrm{~F}_{3 / 2}$ and ${ }^{4} \mathrm{I}_{11 / 2}$

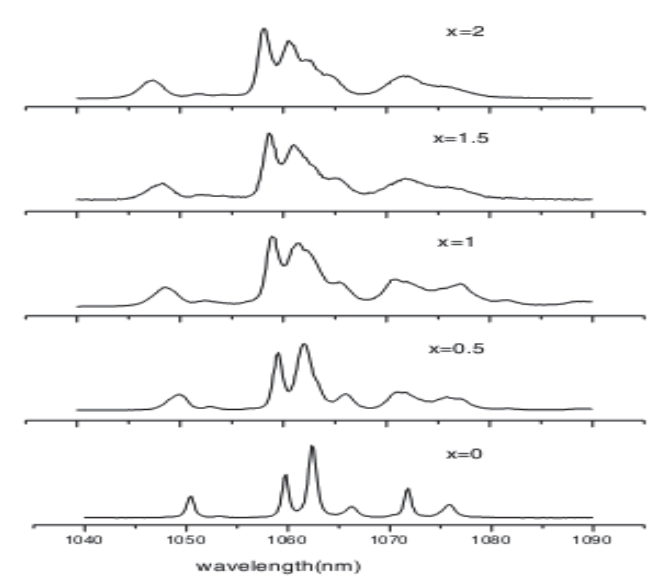

Fig. 30. Fluorescence spectra is influenced by $\mathrm{x}$ in $\mathrm{Nd}: \mathrm{Y}_{3} \mathrm{Sc}_{\mathrm{x}} \mathrm{Al}_{5-\mathrm{x}} \mathrm{O}_{12}$

The Nd:YSAG ceramics disks used in our experiment are made by Shanghai Institute of Ceramics, Chinese Academy of Science. By changing the $x$ in Nd:Y3ScxAl5-xO12, we obtain the fluorescence spectra, as shown in figure 30. The two strongest peaks locate from 
$1060.5 \mathrm{~nm}$ to $1062.5 \mathrm{~nm}$ depending on the concentration of Sc, corresponding to the transition of R1-Y1 and R2-Y3 in in figure 29. Along with the increasing amount of Sc3+, the bandwidth of fluorescence broadens. Moreover, the branching ratio and position of emission peaks also change. We attributed this inhomogeneous line broadening to the expansion of lattice and complex surroundings of $\mathrm{Nd} 3+$ ions in the disordered YSAG lattice.

The schematic diagram of experiment setup is shown in Figure. 31. The size of $\mathrm{Nd}: \mathrm{Y}_{3} \mathrm{Sc}_{1.5} \mathrm{Al}_{3.5} \mathrm{O}_{12}$ ceramics disk is $\varnothing 12 \mathrm{~mm} * 1 \mathrm{~mm}$ with $\mathrm{Nd}^{3+} 4$ at. \% doped and both the surfaces of the sample are coated with antireflection-film at $1064 \mathrm{~nm}$. The laser experiments are carried out at room temperature without active cooling system. A fiber-coupled LD working at the central wavelength of $808 \mathrm{~nm}$ is used as pump source. The fiber core diameter is $200 \mu \mathrm{m}$ with the numerical aperture of 0.22 . By 2 lenses coupling system, the pump beam is focused on the ceramics' surface to produce a pump light footprint of about $60 \mu \mathrm{m}$ in diameter. We apply a plan-plan cavity with an overall length of $\sim 7 \mathrm{~mm}$. The front mirror is antireflection coated at $808 \mathrm{~nm}$ and highly reflecting at $1064 \mathrm{~nm}$. The rear mirror is the planeparallel one as output coupler. A dichroic beam splitter $\left(45^{\circ}\right)$ is used to reflect the laser and filter out the pump light. The laser output characteristics are analyzed for their spectral content and power with an optical spectrum analyzer (YOKOGAWA AQ6370) and a power meter (Spectra-Physics 407A), respectively.

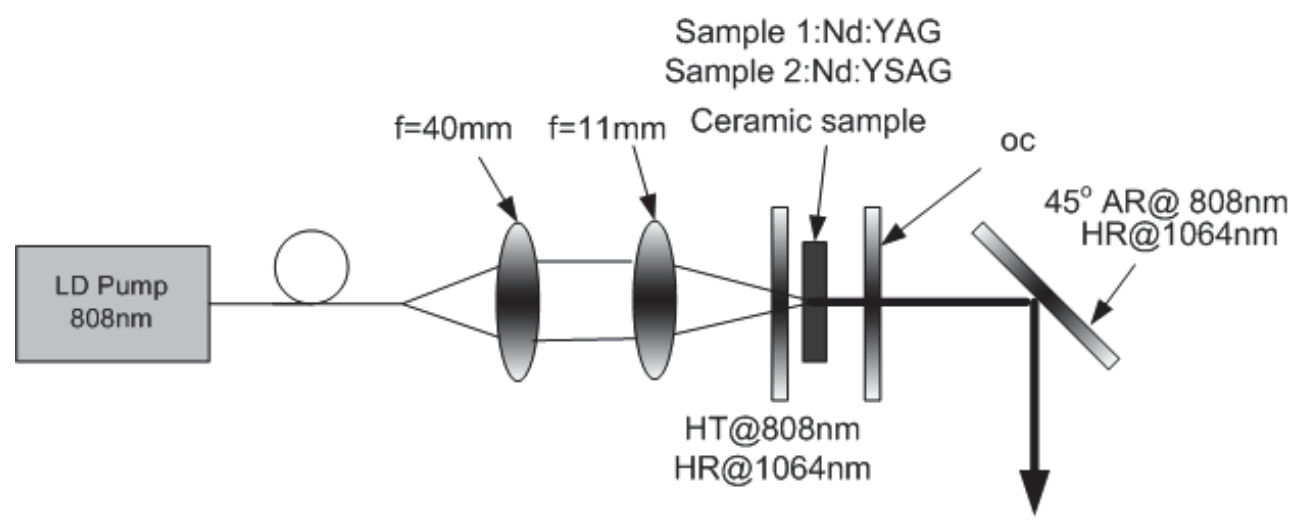

Fig. 31. Schematic diagram of the experimental setup

In the experiment, the ceramics absorbs about $64 \%$ of the pump power set on its surface. The output couplers with different transmission 3\%, 3.9\%, 6\% and 10\% at $1064 \mathrm{~nm}$ are used for laser output experiments respectively. The results are shown in Figure. 32. The laser threshold increases from $0.345 \mathrm{~W}$ to $1.03 \mathrm{~W}$ with the increasing of transmission of the output coupler. The maximum output power of $0.356 \mathrm{~W}$ is achieved at absorbed pumping power of $1.96 \mathrm{~W}$ with the output coupler of $\mathrm{T}_{\mathrm{oc}}=10 \%$. Correspondingly, the optical-optical efficiency is $18.2 \%$ and the slope efficiency is $23.2 \%$. The emission spectra of the laser output is shown in Figure. 33., in which the absorbed pump power is $1.52 \mathrm{~W}$ with the output coupler transmission of $10 \%$. We observes two wavelengths oscillate simultaneously. 


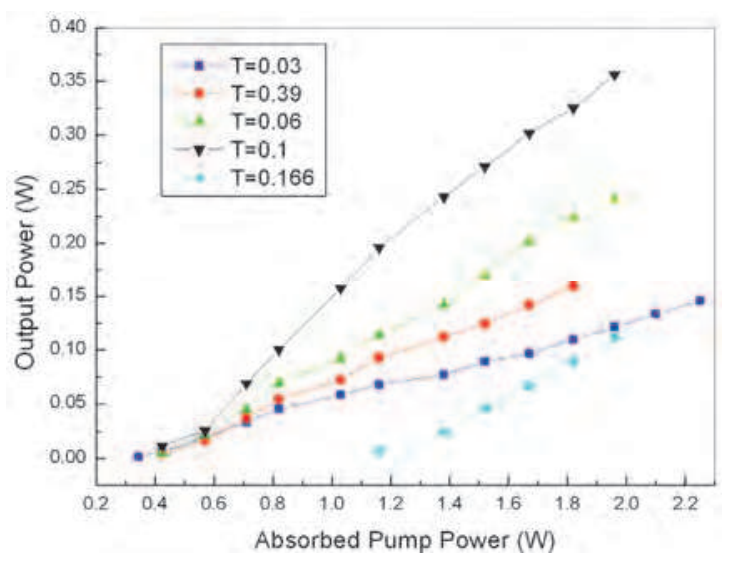

Fig. 32. Output power of the laser emission vs. absorbed pump power for different output couplers

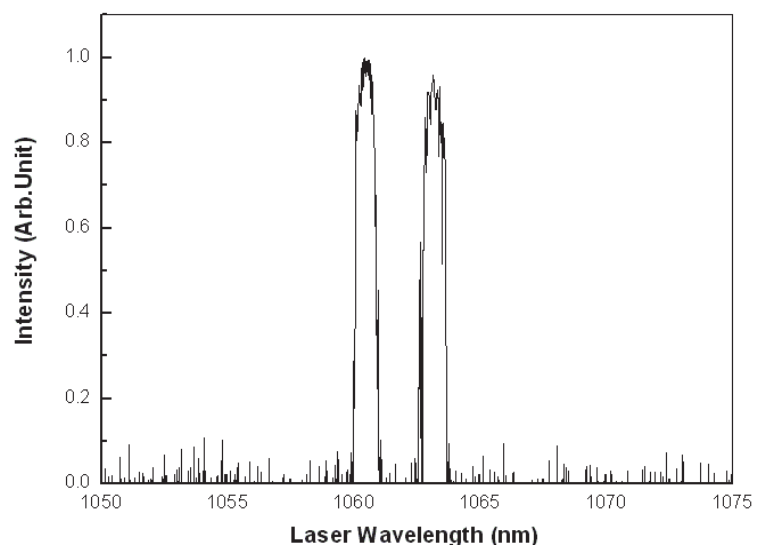

Fig. 33. Emission spectra of the laser: two wavelengths oscillates simultaneously

In order to make a comparison and get more conclusive results, we apply the same experimental environment to another 2 at. \% doped Nd:YAG ceramics disk. The output transmission is $\mathrm{T}_{\mathrm{OC}}=3 \%$. During the experiment, two-wavelengths' oscillation is also observed in Nd:YAG ceramics disk. But the behavior of the two wavelengths in Nd:YAG is totally different with that in Nd:YSAG when the pump power is increasing. In Figure. 34(a), at the absorbed pump power of $0.347 \mathrm{~W}$ which is just above the threshold, only $1064 \mathrm{~nm}$ can oscillate in Nd:YAG. When the pumping intensity enhanced, another wavelength at $1061 \mathrm{~nm}$ appears. Further increasing the pumping power, the intensity of $1061 \mathrm{~nm}$ and $1064 \mathrm{~nm}$ increase synchronously. In Figure. 34 (b), the first laser wavelength operated at $1059.9 \mathrm{~nm}$ in $\mathrm{Nd}$ :YSAG. Increasing the pump power, signal laser at the wavelength of $1063.8 \mathrm{~nm}$ and 1059.9nm radiate form Nd:YSAG simultaneously. Boosting the pumping power, a competitive laser output is shown, in which the intensity of laser at $1059.9 \mathrm{~nm}$ decreases and that of laser at $1063.8 \mathrm{~nm}$ increases. 


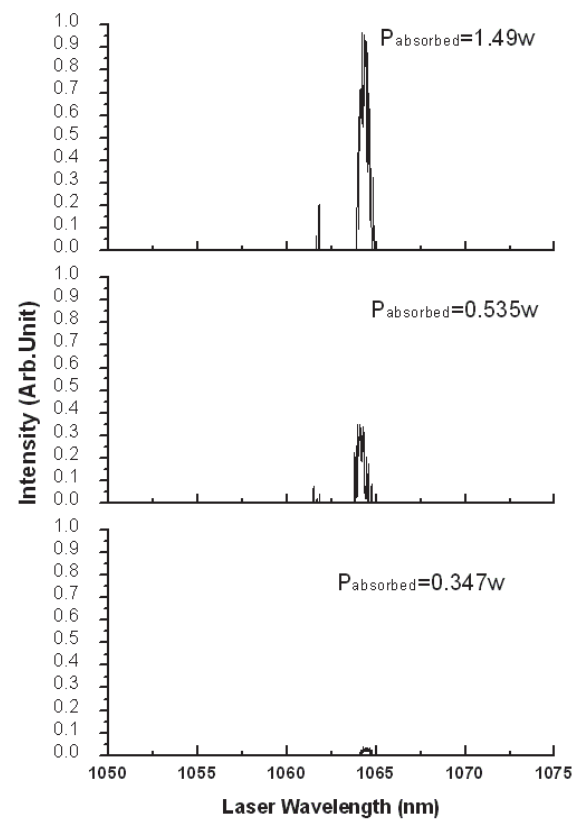

(a) $\mathrm{Nd}: \mathrm{YAG}$

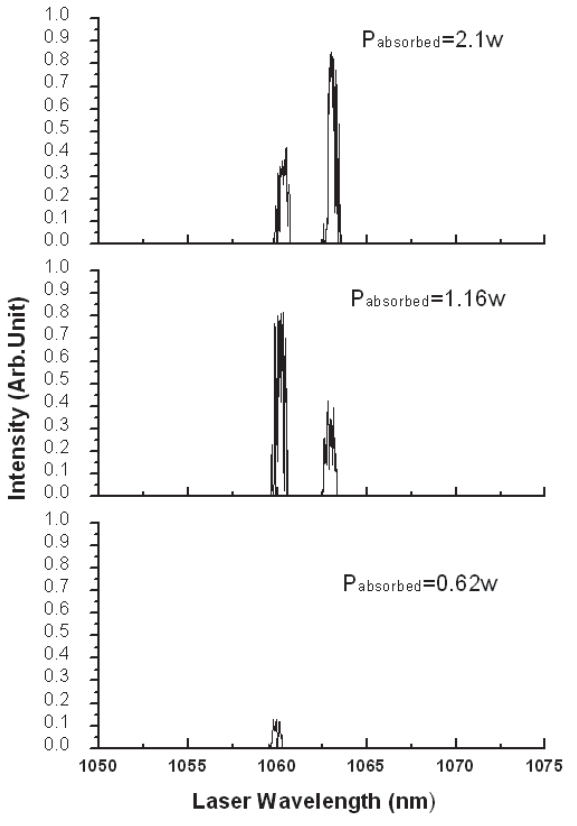

(b) Nd:YSAG

Fig. 34. Different behavior of (a) Nd:YAG and (b) Nd:YSAG along with increasing pump power.

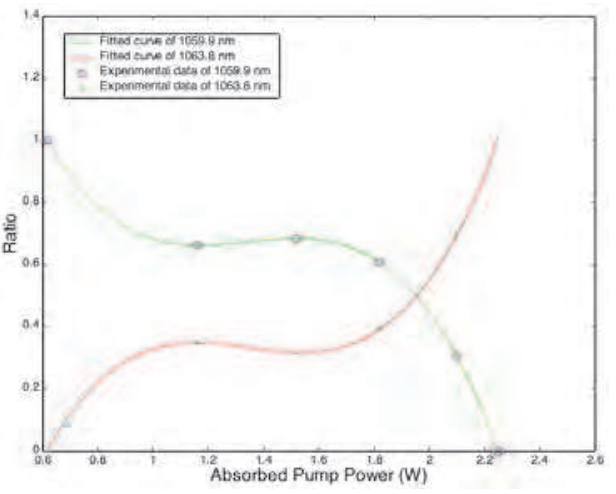

(a)

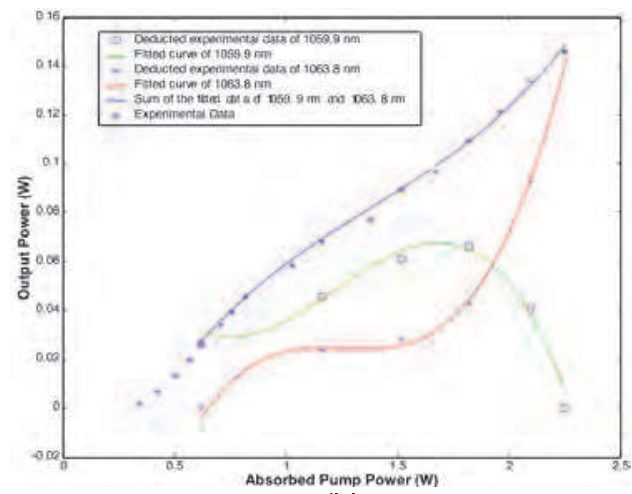

(b)

Fig. 35. Experimental and deducted data of the two wavelengths

By assuming that the total laser output only contains the power of $1059.9 \mathrm{~nm}$ and $1063.8 \mathrm{~nm}$, which ignores noise, we can safely calculate the ratio of each wavelength contributed to the laser output power from the recorded 6 groups of spectrum data for different absorbed pump power. The results are shown in Figure. 35.(a), the ring (o) for 1059.9nm and the plus (+) for $1063.8 \mathrm{~nm}$. The green solid line is polynomial fitting curve for $1059.9 \mathrm{~nm}$, and the red line is for $1063.8 \mathrm{~nm}$. Correspondingly, the output power of each wavelength can be deducted from the 
recorded experimental data for $\mathrm{T}_{\mathrm{oc}}=3 \%$, as shown in Figure. 35. (b). We also work out the fitted functions of the power of $1059.9 \mathrm{~nm}$ and $1063.8 \mathrm{~nm}$. Laser $1059.9 \mathrm{~nm}$ experienced a whole climbing-hill process, in which its power ascended when absorbed pump power is under 1.7W and then descended. On the other hand, laser 1063.8nm exhibited an always-climbing process. The blue line, corresponding to the sum up fitted function, is consistent with the experimental data obtained by power meter marked by start $\left(^{*}\right)$ in $(b)$.

\subsection{Explanation for dual-wavelength output behavior in Nd:YSAG}

While considering the different laser behavior of the two ceramics samples, we attribute these to the different structure of the ceramics. By inviting $\mathrm{Sc}^{3+}$ to the $\mathrm{Nd}: \mathrm{YAG}$ to make $\mathrm{Nd}: Y S A G$, we actually change the structure of the crystal lattice within the ceramics. When the $\mathrm{Sc}^{3+}$ ions (with ion radii larger than $\mathrm{Al}^{3+}$ ions but smaller than $\mathrm{Y}^{3+}$ and $\mathrm{Nd}^{3+}$ ions) enter the lattice, part of the $\mathrm{Al}^{3+}$ will be replaced. But the replacement is random. The difference of ion radii, chemical and physical properties between $\mathrm{Sc}^{3+}$ and $\mathrm{Al}^{3+}$ ions would lead to an almost unpredictable replacing situation. It is highly possible that around the optical center- $\mathrm{Nd}^{3+}$, one site is covered with $\mathrm{Al}^{3+}$ and the other site covered with both $\mathrm{Al}^{3+}$ and $\mathrm{Sc}^{3+}$, as illustrated in Figure.36 . Besides, at different part within the ceramics, $\mathrm{Nd}^{3+}$ are affected by different but similar crystal field, originated from the disorder nature of this new material. Thus the introduction of $\mathrm{Sc}^{3+}$ creates different local environments for the $\mathrm{Nd}^{3+}$ ions which results in multiple sites having different symmetries. The effect of this substitutional disorder is also illustrated: the more $\mathrm{Sc}^{3+}$ enters YAG, the more asymmetric the lattice is, and the more evident the inhomogeneous broadening is presented. Moreover, the transition possibility between different stark levels is also changed. We assume it as multi-sites. The grain boundary within the ceramics material would produce even more complex multi-sites of optical centers, such as $\mathrm{Nd}^{3+}$ ions right at the grain boundary or within a single-crystal grain, for instance.

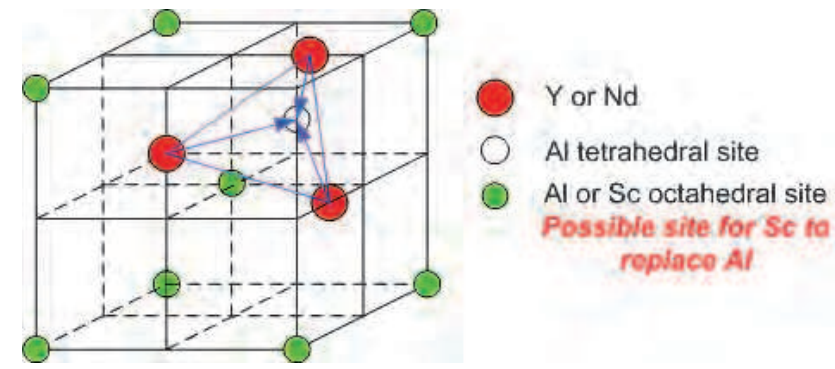

Fig. 36. Positions of $\mathrm{Y} 3+, \mathrm{Al} 3+$ in $\mathrm{YAG}$ lattice: when $\mathrm{Sc} 3+$ enters the lattice, the replacement of $\mathrm{Al} 3+$ in octahedral site is random

In order to give a reasonable explanation of this competitive phenomenon, mutual interactions between ions (instead of isolated ion) are considered and an energy transfer model is applied, as illustrated in Figure.37. When the concentration of active ions is increased, such as in high doped materials, long before the appearance of new lines due to pairs or modifications in radiative transition probabilities, a migration of energy between centers is found. In fact, the energy transfer probability is proportional to the activator concentration [32]:

$$
\mathrm{W}_{\mathrm{t}}=\mathrm{UN}_{\mathrm{A}},
$$

where $U$ is a constant that depends on the type of interaction; $N_{A}$ is the activator concentration. 
Let us consider the simple case of two ions with excited states of different energies, see Figure.37. (a). Then for small energy mismatch (about $100 \mathrm{~cm}^{-1}$ ), energy transfer assisted by one or two phonons can take place [33]. As far as $\mathrm{Nd}: \mathrm{Y}_{3} \mathrm{Sc}_{1.5} \mathrm{Al}_{3.5} \mathrm{O}_{12}$ ceramics is concerned, the fluorescent intensity around $1059 \mathrm{~nm}$ is stronger than that of around $1062.5 \mathrm{~nm}$. Without any control of the output laser, laser at $1059.9 \mathrm{~nm}$ will certainly oscillate first. Meanwhile, when energy transfers from one particular site to another which has slightly different surroundings, the lattice will absorb energy as non-radiative transitions. Thus the "losing of frequency" (lowered energy) leads to the switching to longer wavelength. From the fluorescence spectra, the second highest peak is at around $1062.5 \mathrm{~nm}$. Since the energy mismatch between $R_{1}$ (upper laser state for $1059.9 \mathrm{~nm}$ ) and $R_{2}$ (upper laser state for $1063.8 \mathrm{~nm})$ is small $\left(\sim 82 \mathrm{~cm}^{-1}\right)$, along with the high doping concentration (4 at. \% doped), it is possible that energy transfers from centers (lattice A) which radiate mainly at 1059 . 9nm, to the other centers (lattice B) mainly radiating at $1063.8 \mathrm{~nm}$. In fact, we can assume the $1059.9 \mathrm{~nm}$ center as sensitizer and the $1063.8 \mathrm{~nm}$ center as activator see Figure.37. (a). With the increasing of pumping intensity, more and more transfer would take place. As a result, the $1063.8 \mathrm{~nm}$ reaches its threshold later and forms the second laser. For there is no outside assistance to influence the transfer, it is natural that higher energy from part of active ions is transferred to other different part of ions and emitted photon with lower energy there. The thermal load of the ceramics will enhance such process. That is the reason for the competitiveve output between 1059.9nm and 1063.8nm.

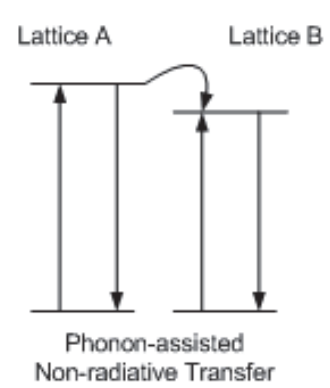

(a)Nd:YSAG

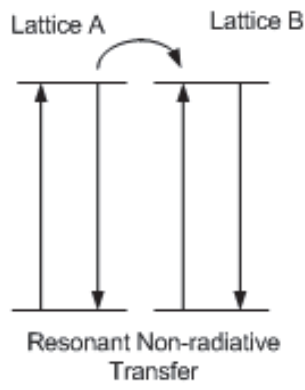

(b)Nd:YAG

Fig. 37. Different energy transfer styles between $\mathrm{Nd}: \mathrm{YAG}$ and $\mathrm{Nd}: \mathrm{YSAG}$

If now we consider another situation: two ions with their nearly equal energy of the excited state, which is the case of $\mathrm{Nd}$ :YAG. Bbecause the $\mathrm{Nd}^{3+}$ ions occupy identical sites in the ordered lattice and the doping concentration is much lower ( 2 at. \% doped), the excitation will jump from one ion (lattice A) to the nearby ion (lattice B) and resulted in almost no energy loss, see Figure. 37. (b). Therefore, with the development of pumping intensity, both the two laser output power increased correspondingly. Again, this proves that the dualwavelength output behavior is the result of Nd:YSAG's own special and complex structure. Terahertz wave attracts many scientists because of its ability to penetrate common materials without harming human tissue like typical X-rays. Several methods are developed to generate it. A very effective way to achieve that is by Difference Frequency Mixing (DFM) of near-IR lasers, usually using 2 seed sources. One of these outstanding jobs is done by Daniel Greeden et al. [9]. Since they used two seed diodes whose wavelengths are 1064.2nm and 1059nm respectively, there is highly possible that this kind of Nd:YSAG ceramics disk can be used to replace the two seed sources in the future. From Figure. 33., when absorbed 
pump power is $1.96 \mathrm{~W}$, the output of $1059.9 \mathrm{~nm}$ and $1063.8 \mathrm{~nm}$ are the same. From this point, utilizing this kind of ceramics laser as seed source to output two near-IR lasers amplified by double-clad fiber laser and then applying DFM methods to generate Terahertz radiation is our future blueprint of a compact Terahertz source.

In our experiment on Nd:YSAG thin disk ceramics, we get $\mathrm{CW}$ laser output and demonstrated the dual-wavelength competitive output phenomenon. By comparing the different laser performance between Nd:YAG and Nd:YSAG and applying an energy transfer model, we discuss and give reasonable explanation for the dual-wavelength competetive output in Nd:YSAG as the disordered replacing of $\mathrm{Al}^{3+}$ ions by $\mathrm{Sc}^{3+}$ ions. This disordered replacing leads to a different energy transfer system in Nd:YSAG. Through the analysis of the behavior of the two wavelengths, we proposed a possible solution to make compact Terahertz source by using one laser source in the future.

\section{References}

[1] Q. Liu, M. Gong, F. Lu, W. Gong, and C. Li, “520-W continuous-wave diode cornerpumped composite Yb:YAG slab laser," Opt. Lett. 30, 726-728 (2005).

[2] R.R. Monchamp, "The distribution coefficient of neodymium and lutetium in Czochralski grown Y3A15O12," J. Cryst. Growth. 11, 310-312 (1971).

[3] "Ceramics YAGs set to challenge single crystals," Opto \& Laser Europe, (2001) http://optics.org/articles/ole/6/12/5

[4] E. Carnall, S. E. Hatch, and W. F. Parsons, “Optical Studies on Hot-Pressed. Polycrystalline CaF2 with Clean Grain Boundaries," Mater. Sci. Res. 3, 165-173 (1966).

[5] A. Ikesue, T. Kinoshita, K. Kamata, and K. Yoshida, "Fabrication and Optical Properties of High-Performance Poolycrystalline Nd:YAG Ceramics for Solid-State Lasers," J. Am. Ceram. Soc. 78, 1033-1040 (1995).

[6] T. Yanagiya, H. Yagi, and A. Ichikawa, "Production of yttrium-aluminum-garnet fine powder," Japanese patent (24 September 1996).

[7] T. Yanagiya, H. Yagi, and H. Yamazaki, "Production of fine power of yttrium aluminum garnet," Japanese patent: (24 September 1996).

[8] Baikowski. Ceramic YAG technical datasheet [EB/OL]. http://www.baikowsku.com/components/pdf/ceramic_yag/YAG_DATA_SHEE T.pdf, 2003. 9.

[9] J. B. Gruber, D. K. Sardar and R. M.Yow, "Energy-level structure and spectral analysis of $\mathrm{Nd} 3+(4 \mathrm{f} 3)$ in polycrystalline ceramic garnet Y3A15O12," J. Appl. Phys. 96, 30503056 (2004).

[10] G. A. Kumar, J. Lu, A. A. Kaminskii, K. Ueda, H. Yagi, T. Yanagitani, and N. V. Unnikrishnan, "Spectroscopic and Stimulated Emission Characteristics of Nd3+ in Transparent YAG Ceramics," IEEE J. Quantum Electron. 40, 747-758 (2004)

[11] J. Lu, M. Prabhu, J. Song, C. Li, J. Xu, K. Ueda, A. A. Kaminshii, H. Yagi, and T. Yanagitani, "Optical properties and highly efficient laser oscillation of Nd:YAG ceramics," Appl. Phys. B 71, 469-473 (2000).

[12] J. Lu, J. Song, M. Prabhu, J. Xu, K. Ueda, and H. Yagi, “High-power Nd: $\mathrm{Y}_{3} \mathrm{Al}_{5} \mathrm{O}_{12}$ Ceramic Laser," Jpn. J. Appl. Phys. 39, 1048-1050 (2000).

[13] J. Lu, T. Murai, K.Takaichi, T. Uematsu, K. Misawa, M. Prabhu, and J. Xu, “72 W $\mathrm{Nd}: \mathrm{Y}_{3} \mathrm{Al}_{5} \mathrm{O}_{12}$ ceramic laser," Appl. Phys. Lett. 78, 3586-3588 (2001).

[14] J. Lu, M. Prabhu, K. Ueda, H. Yagi, T. Yanagitani, A. Kudryashov, and A. A. Kaminshii, "Potential of Ceramic YAG Lasers," Laser. Phys. 11, 1053-1057 (2001). 
[15] J. Lu, K. Ueda, H. Yagi, T. Yanagitani, Y. Akiyama, and A. A. Kaminshii, “Neodymium doped yttrium aluminum garnet (Y3Al5O12) nanocrystalline ceramics- - a new generation of solid state laser and optical materials," J. Alloy. Compd. 341, 220-225 (2002).

[16] J. Lu1,*, M. Prabhu1, J. Song1, C. Li1, J. Xu1, K. Ueda1, A.A. Kaminskii2, H. Yagi3, T. Yanagitani3, Optical properties and highly efficient laser oscillation of Nd:YAG ceramics, Appl. Phys. B 71, 469-473 (2000) / Digital Object Identifier (DOI) $10.1007 /$ s003400000394

[17] W. Koechner, Solid State Laser Engineering, 40-41(2002)。

[18] Xiaodan Wang , Xiaodong Xu, Xionghui Zeng, Zhiwei Zhao,Benxue Jiang , Xiaoming He, Jun $\mathrm{Xu}$; Effects of $\mathrm{Yb}$ concentration on the spectroscopic properties of $\mathrm{Yb}$ : $\mathrm{Y} 3 \mathrm{Al} 15 \mathrm{O} 12$

[19] Xiaodong $\mathrm{Xu}$, Zhiwei Zhao, PingXin Song, Jun $\mathrm{Xu}$, Peizhen Deng ; Growth of highquality single crystal of 50 at.\% Yb:YAG and its spectral properties ; Journal of Alloys and Compounds 364, 311-314(2004)

[20] Takunori Taira, William M.Tulloch, and Robert L.Byer, Modeling of quasi-three-level lasers and operation of CW Yb:YAG lasers, Applied Optics,36(1997)

[21] S.Belogurov, G.Bressi,et al,Properties of Yb-doped scintillators:YAG,YAP,LuAG, Nuclear Instruments and Methods in Physics Research A 516,58-67(2004)

[22] Tsunekane, Masaki; Taira, Takunori, Optics Letters, 31(13),2003-2005 (2006)

[23] Haiwu Yu, Gilbert Bourdet, Applied Optics,44, 7161(2005)

[24] D.S.Sumida, T.Y.Fan,Optics Letters, 19(17),1343(1994)

[25] J. Lu, H. Yagi, K. Takaichi, T. Uematsu, J.F. Bisson, Y. Feng, A.Shirakawa, K. Ueda, T. Yanagitani, and A.A. Kaminskii, "110W ceramic Nd3+:Y3A15O12 laser," Appl. Phys.B. 79 (2004)25-28.

[26] http://www.llnl.gov/nif/psa/sshcl.html

[27] Tao Feng, Jianlin Shi, Jiyang Chen, and Danyu Jiang, "Fluorescence emission enhancement of transparent Nd:YSAG ceramics by Sc2O3 doping," J. Opt. Soc. Am. B. 22(2005)2134-2137.

[28] Tao Feng, "Synthesis and greatly enhanced fluorencence emission of transparent Nddoped Y3ScxA15-xO12 ceramic," J. Mater. Res. 20(2005)2322-2327.

[29] Yoichi Sato, Jiro Saikawa , Takunori Taira , and Akio Ikesue.,"Characteristics of Nd3+doped Y3ScA14O12 ceramic laser," Optical Material .29(2007)1277-1282.

[30] J.Lu, K.Takaichi, T.Uematsu, A.Shirakawa, M.Musha, and K.Ueda, "Promising ceramic laser material: Highly transparent Nd3+:Lu2O3 ceramic," Appl. Phys. Lett.. 81(2002)4324-4326.

[31] John B.Gruber, Marian E. Hills, Toomas H.Allik, C.K.Jayasankar, John R. Quagliano, and F.S.Richardson "Compararive analysis of $\mathrm{Nd} 3+(4 \mathrm{f} 3)$ energy levels in four garnet hosts," Physical Review B. 41 (1990)7999-8012.

[32] Daniel Creeden, John C, McCarthy, Peter A.Ketteridge, Peter G.Schunemann, Timothy Southward, James J.Komiak, and Evan O.Chicklis, "Compact,high average power,fiber-pumped terahertz source for active real-time imaging of concealed objects," Optics Express.15(2007)6478-6483.

[33] F.Auzel and R.Hull, Spectroscopic Properties of Rare Earths in Optical Materials, Chap. 5 , Tsinghua University Press and Springer-Verlag Berlin Heidelberg, 2005. 


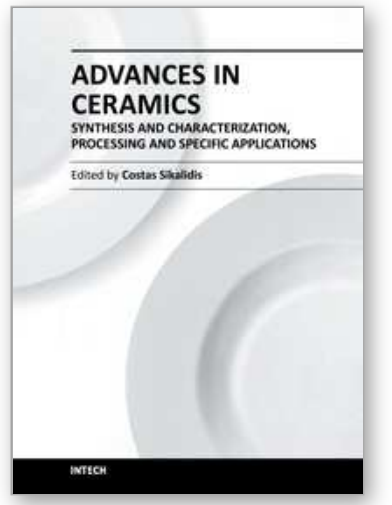

\author{
Advances in Ceramics - Synthesis and Characterization, \\ Processing and Specific Applications \\ Edited by Prof. Costas Sikalidis
}

ISBN 978-953-307-505-1

Hard cover, 520 pages

Publisher InTech

Published online 09, August, 2011

Published in print edition August, 2011

The current book contains twenty-two chapters and is divided into three sections. Section I consists of nine chapters which discuss synthesis through innovative as well as modified conventional techniques of certain advanced ceramics (e.g. target materials, high strength porous ceramics, optical and thermo-luminescent ceramics, ceramic powders and fibers) and their characterization using a combination of well known and advanced techniques. Section II is also composed of nine chapters, which are dealing with the aqueous processing of nitride ceramics, the shape and size optimization of ceramic components through design methodologies and manufacturing technologies, the sinterability and properties of $\mathrm{ZnNb}$ oxide ceramics, the grinding optimization, the redox behaviour of ceria based and related materials, the alloy reinforcement by ceramic particles addition, the sintering study through dihedral surface angle using AFM and the surface modification and properties induced by a laser beam in pressings of ceramic powders. Section III includes four chapters which are dealing with the deposition of ceramic powders for oxide fuel cells preparation, the perovskite type ceramics for solid fuel cells, the ceramics for laser applications and fabrication and the characterization and modeling of protonic ceramics.

\title{
How to reference
}

In order to correctly reference this scholarly work, feel free to copy and paste the following:

Qihong Lou, Jun Zhou, Yuanfeng Qi and Hong Cai (2011). Laser Applications of Transparent Polycrystalline Ceramic, Advances in Ceramics - Synthesis and Characterization, Processing and Specific Applications, Prof. Costas Sikalidis (Ed.), ISBN: 978-953-307-505-1, InTech, Available from:

http://www.intechopen.com/books/advances-in-ceramics-synthesis-and-characterization-processing-andspecific-applications/laser-applications-of-transparent-polycrystalline-ceramic

\section{INTECH}

open science | open minds

\author{
InTech Europe \\ University Campus STeP Ri \\ Slavka Krautzeka 83/A \\ 51000 Rijeka, Croatia \\ Phone: +385 (51) 770447 \\ Fax: +385 (51) 686166 \\ www.intechopen.com
}

\author{
InTech China \\ Unit 405, Office Block, Hotel Equatorial Shanghai \\ No.65, Yan An Road (West), Shanghai, 200040, China \\ 中国上海市延安西路65号上海国际贵都大饭店办公楼405单元 \\ Phone: +86-21-62489820 \\ Fax: +86-21-62489821
}


(C) 2011 The Author(s). Licensee IntechOpen. This chapter is distributed under the terms of the Creative Commons Attribution-NonCommercialShareAlike-3.0 License, which permits use, distribution and reproduction for non-commercial purposes, provided the original is properly cited and derivative works building on this content are distributed under the same license. 